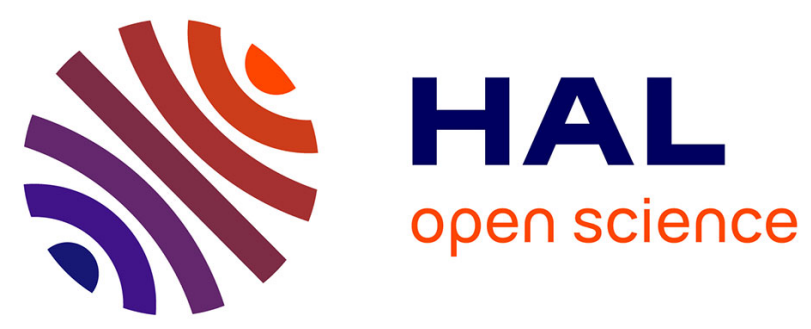

\title{
Le Rhône et la Saône à Lyon à l'époque romaine: bilan archéologique
}

\author{
Armand Desbat, Jean-Paul Lascoux
}

\section{To cite this version:}

Armand Desbat, Jean-Paul Lascoux. Le Rhône et la Saône à Lyon à l'époque romaine: bilan archéologique. Gallia - Archéologie de la France antique, 1999, Le Rhône romain, 56, pp.45-69. 10.3406/galia.1999.3243 . hal-01912997

\section{HAL Id: hal-01912997 \\ https://hal.science/hal-01912997}

Submitted on 16 Jan 2020

HAL is a multi-disciplinary open access archive for the deposit and dissemination of scientific research documents, whether they are published or not. The documents may come from teaching and research institutions in France or abroad, or from public or private research centers.
L'archive ouverte pluridisciplinaire HAL, est destinée au dépôt et à la diffusion de documents scientifiques de niveau recherche, publiés ou non, émanant des établissements d'enseignement et de recherche français ou étrangers, des laboratoires publics ou privés.

\section{(ㅇ)(1) $\$$}

Distributed under a Creative Commons Attribution - NonCommercial - NoDerivatives| 4.0 


\title{
LE Rhône ET LA SAÔNE À LYON À L'ÉPOQUE ROMAINE
}

\section{Bilan archéologique}

\author{
Armand Desbat et Jean-Paul Lascoux
}

\begin{abstract}
Mots-clés. Lyon, Lugdunum, Rhône, Saône, confluent, presqu'île, quartiers fluviaux.
Key-words. Lyon, Lugdunum, Rhône, Saône, confluence, peninsula, riverside districts.

Résumé. Depuis une vingtaine d'arnées, plus de quarante opérations archéologiques ont concerné non seulement la presqu'île mais encore les quartiers fluviaux de Lyon, comme Saint-jean ou Vaise. Grâce à ces fouilles et aux travaux des géomorphologues, la question du confluent, de même que l'évolution des quartiers de la ville basse entre la période augustéenne et le IV's. commencent à être mieux appréhendés.
\end{abstract}

\begin{abstract}
Since twenty years, more than forty archaeological rescues have been done inside the peninsula and also along the riverside districts of Lyon such as Sainl-Jean or Vaise. Thanks to these excavations and to the research work of geomorphologists, the question of the confluent as well as the evolution of the districts of the lower part of the town, from the Augustan period to the $4^{\text {th }}$ century, is now better known.
\end{abstract}

La presqu'île et les berges de la Saône ont été, depuis des siècles, le lieu de nombreuses découvertes archéologiques. Malheureusement la documentation sur ces découvertes est pratiquement inexistante et notre connaissance se limite le plus souvent à de simples mentions dans Lyon souterrain... d'Artaud (1846) ou dans $L a$ nouvelle histoire de Lyon de Steyert (1895). Reconstruire l'histoire de la presqu'île et son évolution morphologique à partir de ces données était une entreprise quasiment impossible. Malgré cela, plusieurs tentatives ont été faites par le passé pour reconstituer la topographie de la presqu'île et surtout le confluent qui constituait une préoccupation majeure des archéologues lyonnais. Cette question revêtait en effet une grande importance aux yeux de nos prédécesseurs, moins pour elle-même que pour la localisation du sanctuaire fédéral situé " ad confluentem».

\section{HYPOTHĖSES ANCIENNES SUR LE CONFLUENT}

L'idée que la presqu'île avait à l'époque romaine une configuration différente de celle qu'on lui connaissait avant les travaux de Perrache à la fin du XVIII's. n'est pas nouvelle. Déjà le R. P. Menestrier (1696) avait envisagé que celle-ci était constituée de plusieurs îles. Depuis, dans la première moitié du XIX ${ }^{\mathrm{e}}$ s., bien d'autres hypothèses ont été émises, qui, presque toutes, supposaient l'existence d'un ou de plusieurs bras vifs, voire de canaux traversant la presqu'île.

Ainsi, dès le début du XIX ${ }^{e}$ s., Chenavard nous propose-t-il, dans la première carte archéologique établie d'après les données d'Artaud, une presqu'île traversée par trois canaux : le premier à la hauteur des Terreaux, le deuxième à la hauteur de la rue Grenette, le troisième 


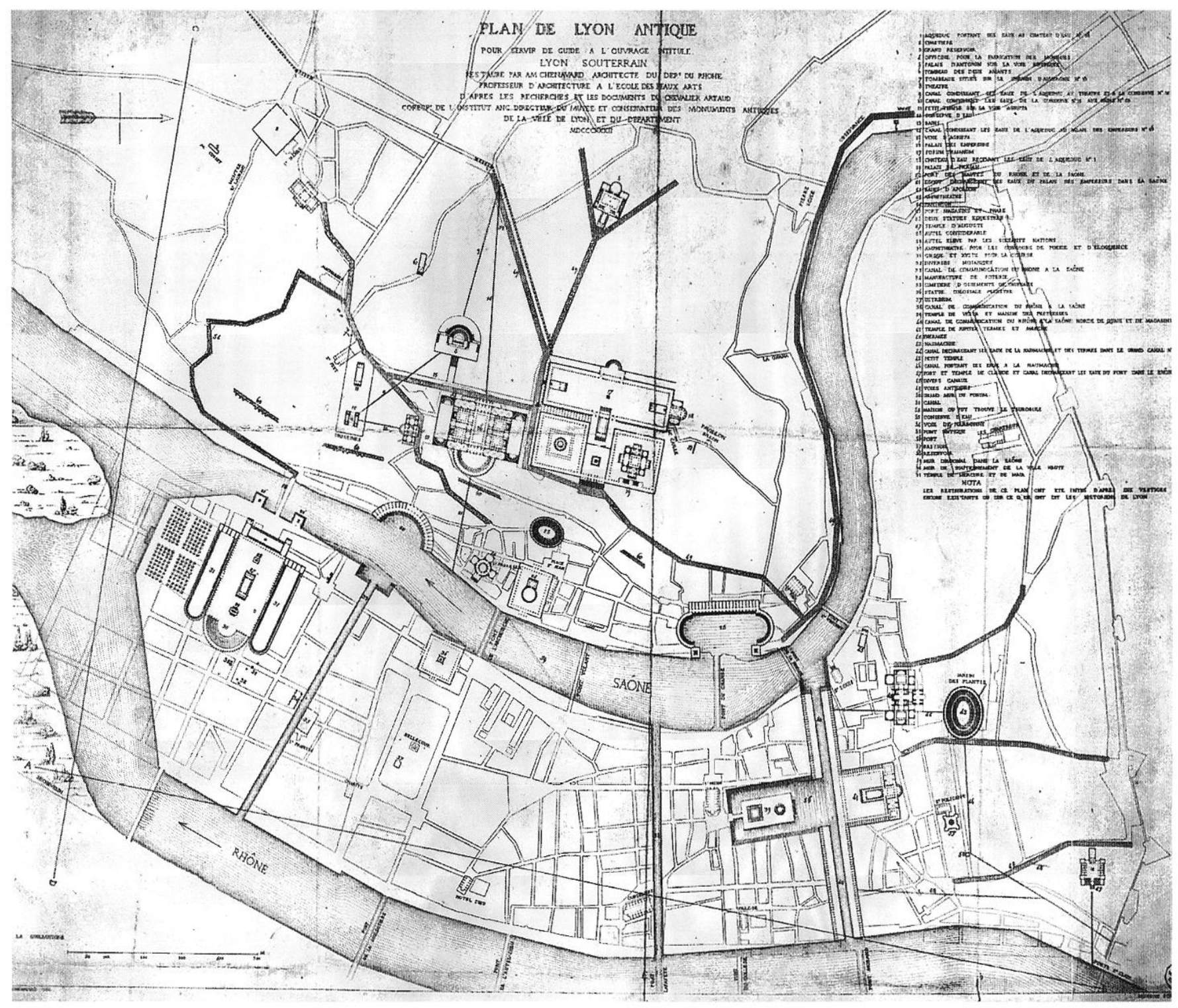

Fig. 17 - Plan de Lyon antique dessiné par A.-M. Chenavard en 1832 destiné à illustrer "Lyon souterrain... " de F.-M. Artaud, 1846 (Chenavard, 1850).

enfin à la hauteur de la rue Sainte-Hélène (Chenavard, 1850) (fig. 17). Cette hypothèse, qui reposait sur l'observation de niveaux d'alluvions et de vides sanitaires d'amphores identifiés à tort comme des épaves, donnait l'image d'une presqu'île fortement structurée par les Romains.

Par la suite, plusieurs historiens et archéologues ont émis d'autres hypothèses s'appuyant, pour une grande partie, sur les vestiges signalés par Artaud, mais aussi sur les textes du Haut Moyen Âge, relatifs à des crues. À la suite de Chenavard, Martin-Daussigny (1853) proposa également une presqu'île morcelée en quatre îles par plusieurs canaux : le premier au Terreaux, le deuxième à la hauteur de la rue Grenette et de la rue Dubois, le troisième aux Jacobins et le quatrième, enfin, au niveau des rues Sala et Sainte-Hélène. Toutefois, il estimait qu'il ne s'agissait pas de confluents mais de « canaux naturels agrandis pour les besoins du commerce » en considérant qu'il n'existait qu'un seul vrai confluent à Ainay ${ }^{21}$.

21. Martin-Daussigny réfute l'idée que le terme de « confluentes " puisse désigner plusieurs confluents, mais estime qu'il sous-entend " aquas». 


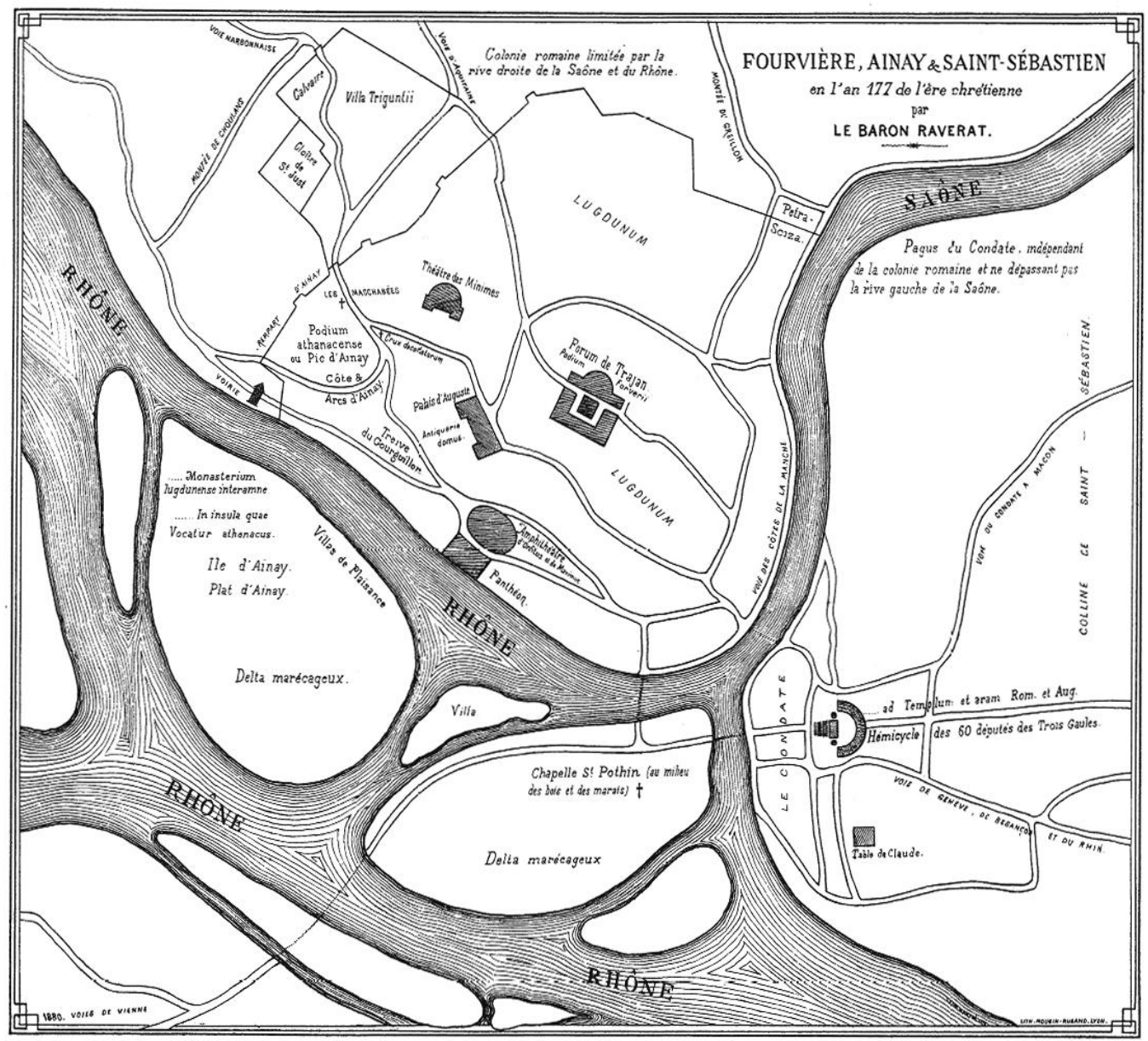

Fig. 18 - Le confluent $\grave{a}$ l'époque romaine selon le baron Raveral (1880).

Cette hypothèse fut fermement combattue par Boissieu (1864), partisan de l'autel fédéral et de l'amphithéâtre à Ainay. En s'appuyant précisément sur les vides sanitaires d'amphores repérés en différents points de la presqu'île, il émit l'idée que celle-ci présentait à l'époque romaine une physionomie finalement voisine de ce qu'elle était à l'époque moderne ${ }^{22}$. Il ne fut pourtant jamais suivi dans cette voie.

Il s'appuie pour cela sur la comparaison avec Coblenz où le terme " confluentes" est employé pour désigner le confluent de la Moselle et du Rhin. "Au temps de la fondation par Plancus, le terrain de la Presqu'île où est aujourd'hui Lyon se divisait à peu près en quatre îles plus ou moins grandes et plus ou moins élevées au-dessus des eaux " (Martin-Daussigny, 1853, p. 4).

22. "Pendant l'ère romaine le confluent n'était pas aux Terreaux, le canal de ce nom est une œuvre du Moyen Âge. Les autres confluents sont de pure imagination ; et des invasions temporaires, des courants successivement formés et comblés par les crues et les inondations, dcs délaissćs plus ou moins persistants, des parties basses souvent marécageuses ne peuvent pas s'appeler un confluent; le grand
Le baron Raverat (1880), critiquant les hypothèses de ses prédécesseurs, proposa de localiser le sanctuaire sur le coteau de Saint-Sébastien tout en restituant de nouveau la presqu'île occupée par plusieurs îles (fig. 18).

Bien que Vermorel (1881) ait réfuté avec des arguments de poids ${ }^{23}$ l'hypothèse d'un bras du Rhône ou d'un canal à l'emplacement des Terreaux, celle-ci fut reprise de nouveau par Allmer et Dissard (1889). Ces

Rhône n'était pas là ; ce qu'on a pris pour le lit de nos fleuves n'est que le niveau de leurs bords à cette époque; enfin ni l'autel d'Auguste ni l'abbaye d'Ainay n'ont été élevés dans une île mais tous les deux, d'après les textes inscrits sur la pierre et le velin, étaient situés inter amnes, ad confluentes Araris et Rhodani " (Boissieu, 1864, p. 51).

23. Vermorel souligne entre autre que, "Au droit des Terreaux, l'étiage du Rhônc est à 1 mètre 90 centimètres plus haut que celui de la Saônc. lec canal aurait donc traversé la faible distance d'un fleuve à l'autre soit environ 650 mètres avec une chute d'eau de 1 mètre 90 . Avec quelle violence indomptable les eaux se seraient précipité par cette issue !» (Vermorel, 1881, p. 8). 


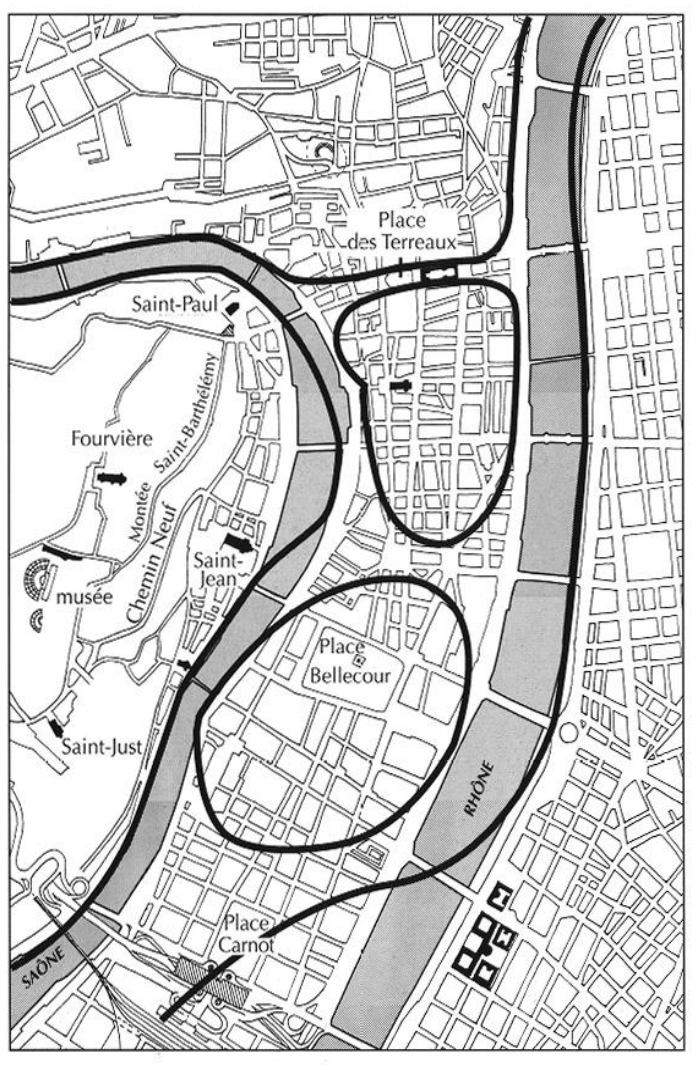

Fig. 19 - Le confluent à l'époque romaine selon A. Allmer et P. Dissard (1889) d'après A. Desbat (1982).

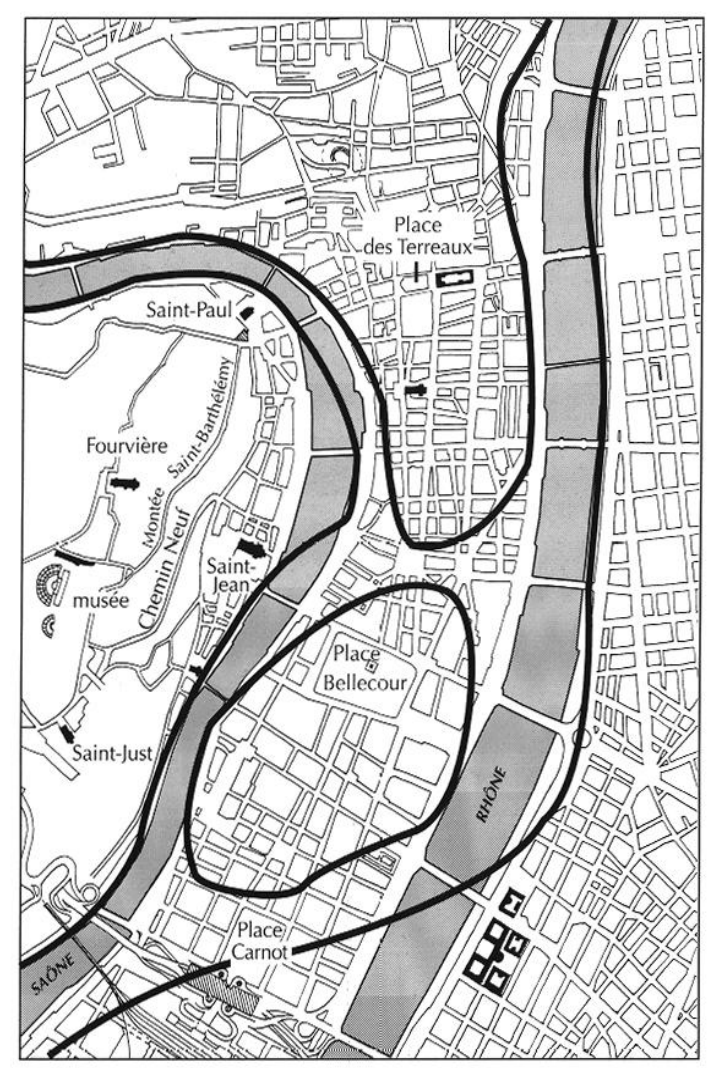

Fig. 21 - Le confluent à l'époque romaine selon M. Audin (1919).

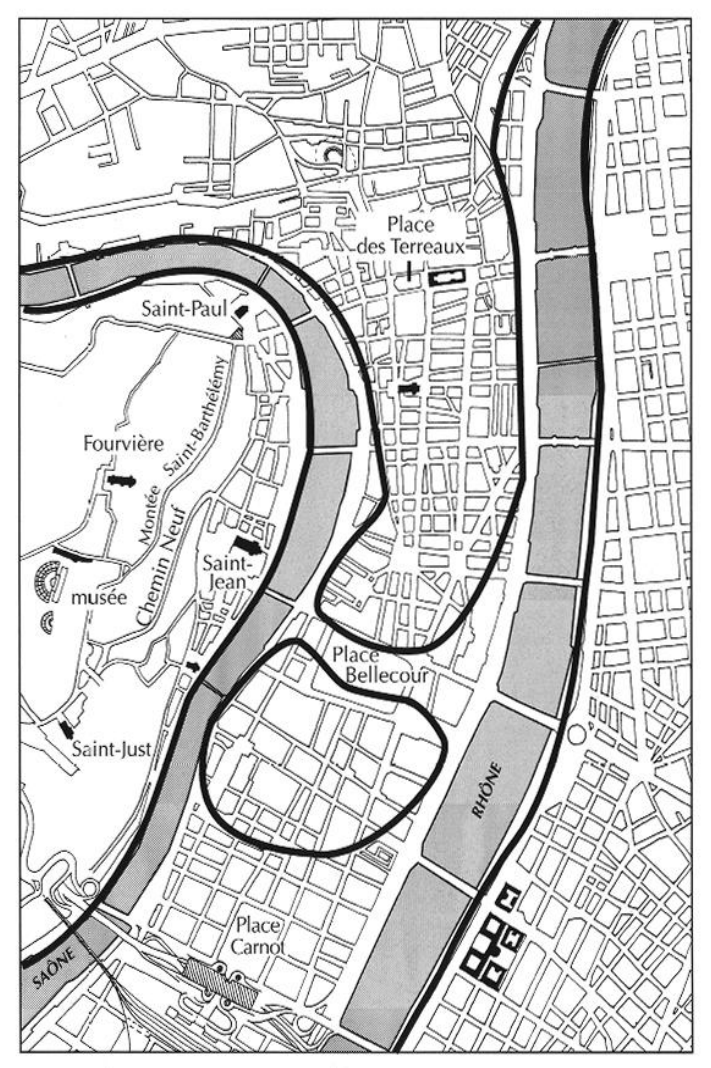

Fig. 20 - Le confluent à l'époque romaine selon A. Steyert (1895).

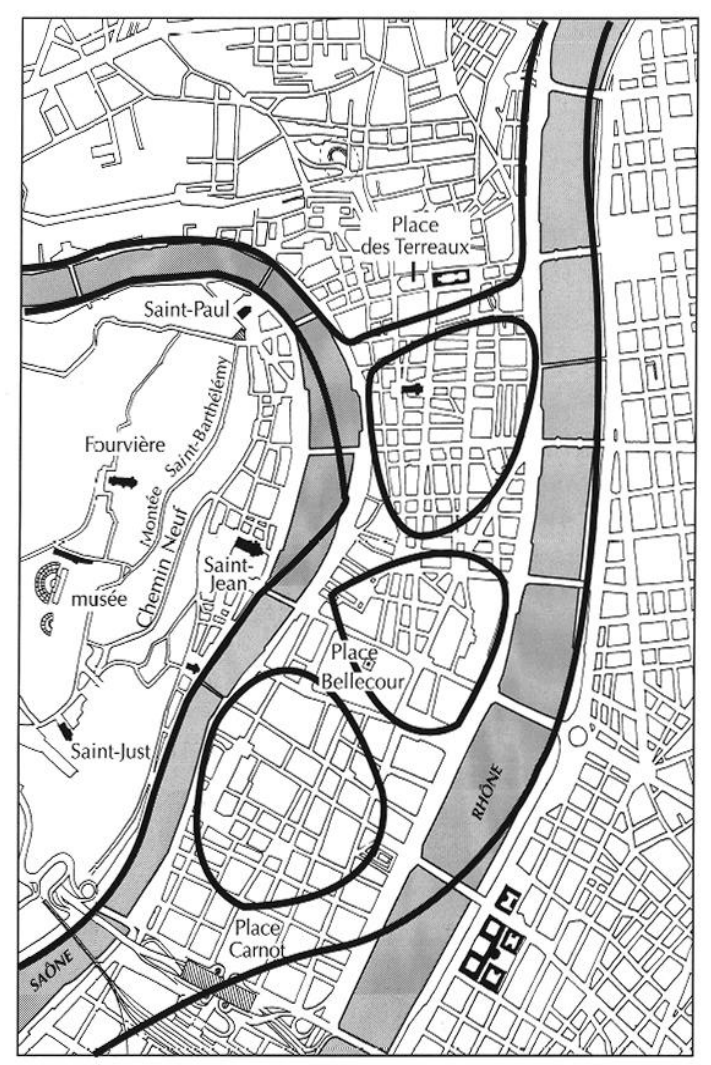

Fig. 22 - Le confluent à l'époque romaine selon C. Germain de Montauzan (1924). 
derniers, tout en conservant un canal artificiel reliant le Rhône et la Saône à la hauteur des Terreaux, ont situé le confluent proprement dit plus au sud, au niveau de la place des Cordeliers, en supposant un bras du Rhône traversant la presqu'île en oblique à la hauteur de SaintBonaventure (fig. 19).

Quelques années plus tard Steyert (1895) critiqua à son tour l'hypothèse du canal des Terreaux et situa le confluent à la hauteur de la place Bellecour (fig. 20), en s'appuyant sur l'existence d'une dépression à la hauteur de la place Le Viste (Steyert, 1895, p. 283, fig. 328).

Marius Audin, reprenant la question en 1919, a formulé quant à lui une hypothèse, souvent reprise par la suite, qui réfutait l'existence d'un canal aux Terreaux, mais supposait la présence d'un large bras du Rhône (et non plus de la Saône) traversant la presqu'île à la hauteur de la place des Jacobins (fig. 21).

Une autre hypothèse fut toutefois défendue peu de temps après par Germain de Montauzan (1924), qui non seulement reprit l'idée d'un canal aux Terreaux, cette fois-ci plus au sud, à l'emplacement de la rue de l'ArbreSec, mais encore supposa l'existence de trois îles et quatre confluents en rajoutant un bras supplémentaire à travers la place Bellecour ${ }^{24}$ (fig. 22).

Enfin, A. Audin, reprenant la question en 1947, puis en 1956, dans son Essai sur la topographie de Lugdunum, réactualisa en la modifiant légèrement l'hypothèse de 1919 et proposa l'existence d'un bras du Rhône à la hauteur des Jacobins, créant ainsi une grande île, identifiée aux Canabées (Audin, 1947, 1956, 1964, 1979) (fig. 23). Cette dernière conjecture, consacrée par l'usage, s'est imposée jusqu'à une date récente, faute d'une lecture critique.

Toutes ces hypothèses avaient en commun de supposer une configuration de la presqu'île antique différente de ce qu'elle pouvait être au Moyen Âge, mais surtout d'en donner une image figée durant toute la période romaine, sans envisager qu'elle ait pu connaître une évolution entre les débuts de l'Empire et les $\mathrm{III}^{\mathrm{e}}$ et $\mathrm{IV}^{\mathrm{e}} \mathrm{s}$. En 1967, l'aménagement d'un grand parking souterrain sous la place Bellecour, de même que le percement

24. Germain de Montauzan suppose les dcux îles du nord « guère habitées, si l'on en juge par leur dénûment total en fait de vestiges antiques signalés, à part quelques inscriptions découvertes presque exclusivement au voisinage de l'église Saint-Pierre. "

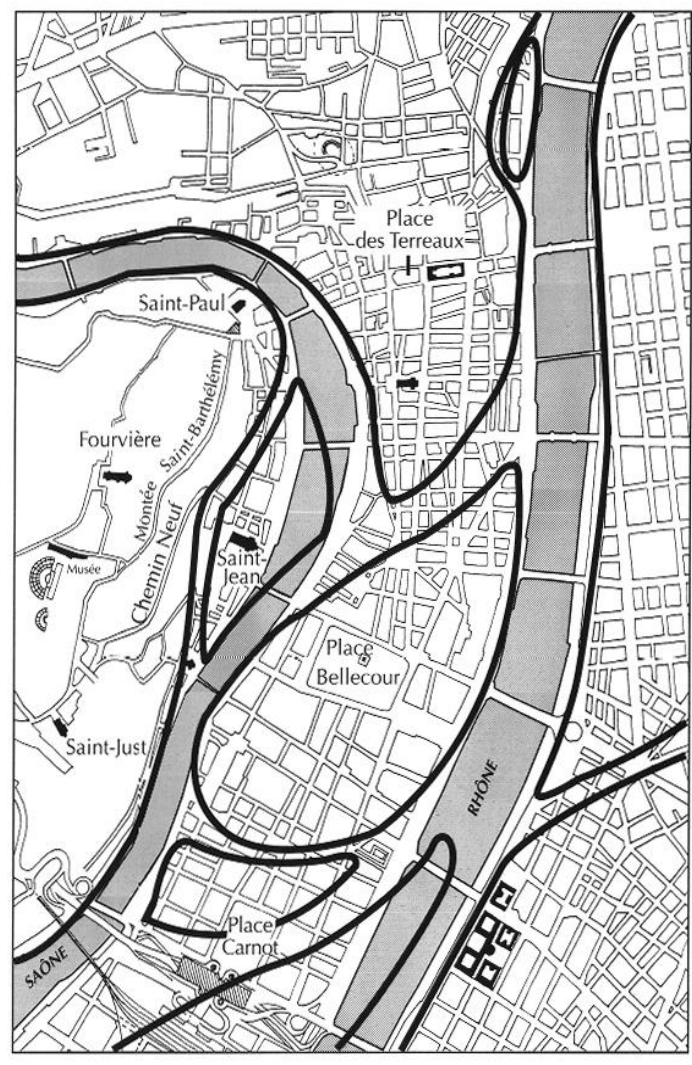

Fig. 23 - Le confluent à l'époque romaine selon A. Audin (1947, 1956, 1964, 1979).

en 1975 de la première ligne du métropolitain furent l'occasion de quelques observations malheureusement trop partielles.

Depuis 1978, une quarantaine d'opérations d'ampleur variée ont été réalisées majoritairement dans la presqu'île, mais aussi sur la rive droite de la Saône, dans le défilé de Pierre-Scize et la plaine de Vaise ( $c f$. annexe I, p. 60-66) (fig. 24).

Notre connaissance est encore très partielle mais il est possible aujourd'hui de dresser un bilan des acquis récents, de confronter ces résultats aux hypothèses émises antérieurement et de dégager, avec beaucoup de prudence, quelques hypothèses nouvelles sur l'évolution de la presqu'île et des quartiers fluviaux.

\section{LA PRESQU'ÎLE}

Depuis 1978, plus de vingt interventions ont été réalisées ; mais c'est surtout depuis une quinzaine d'années 


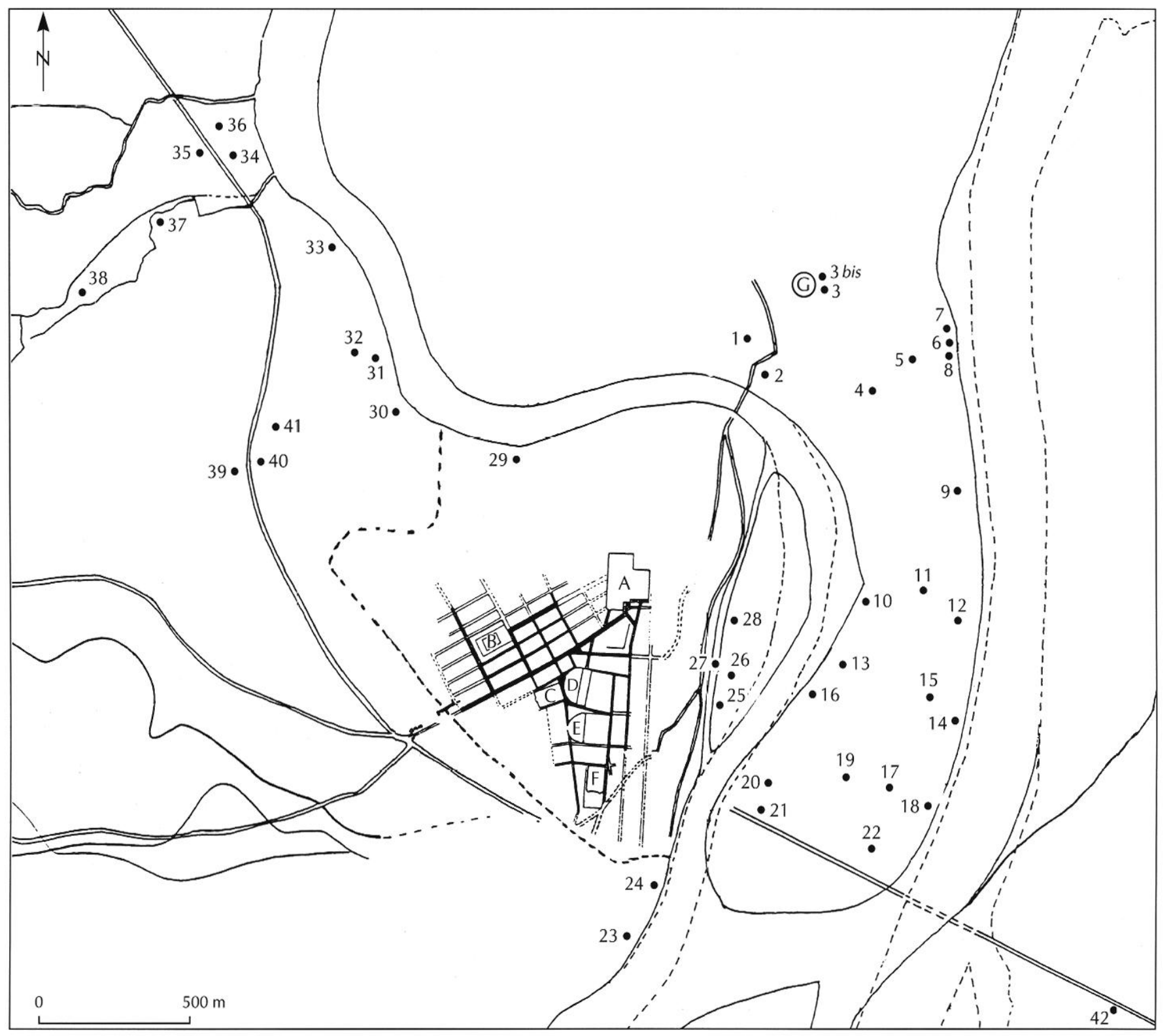

Fig. 24 - Carte des interventions archéologiques réalisées depuis 1978 dans la presqu île et les quartiers fluviaux (les $n^{\text {os }}$ renvoient à ceux de l'annexe I) : A, forum supposé; $B$, sanctuaire du culte impérial; $C$, sanctuaire de Cybèle; $D$, théâtre; $E$, odéon; $F$, grands thermes ;

$G$, amphithéâtre.

que la multiplication des opérations de fouilles de sauvetage dans la presqu'île a fourni un grand nombre d'informations nouvelles. Celles-ci portent essentiellement sur la partie centrale, comprise entre la place Bellecour et les Terreaux, alors que les découvertes de la fin du $\mathrm{XIX}^{\mathrm{e}} \mathrm{s}$. avaient surtout concerné la partie méridionale, au sud de Bellecour, où de nombreuses mosaïques furent exhumées.

Notre connaissance de la presqu'île a considérablement progressé grâce à ces nombreuses opérations mais aussi grâce au travail des géomorphologues sous la direction de J.-P. Bravard ${ }^{25}$.

25. L'intérêt des travaux géomorphologiques est de proposer un modèle du confluent et de son évolution à l'époque antique indépendamment de toutes références, notamment à l'emplacement du temple de Rome et d'Auguste et à la forme de la presqu'île au Moyen Âge et à l'ćpoque moderne. Par ailleurs, la confrontation de ce modèle et des données archéologiques conduit à préciser le rapport entre les formes de l'occupation et le milieu, et à mieux définir ainsi le phénomène urbain et ses composantes. 
Le problème du confluent commence à être appréhendé de manière cohérente ${ }^{26}$. Il ressort des diverses observations réalisées à travers les fouilles récentes qu'il n'existe pas ou plus de bras actifs traversant la presqu'île à l'époque romaine, contrairement à ce qu'avançaient nos prédécesseurs. En effet, les vides sanitaires et les remblais ne sont pas installés dans le courant, mais dans des zones inondables en cours d'assainissement. Néanmoins, l'existence de nombreux chenaux de tressage et la péjoration climatique observée entre la fin du I ${ }^{\text {er }}$ s. avant J.-C. et la fin du siècle suivant (Bravard et al., 1997) ont constitué une entrave à l'urbanisation comme en témoignent l'alternance d'occupations et de dépôts d'inondation, ainsi que le colmatage progressif, naturel ou anthropique, des microchenaux hérités de l'Âge du Fer ${ }^{27}$ (cf. annexe I : place des Célestins $n^{\circ} 13$, p. 63 et place de la Bourse $n^{\circ} 9$, p. 62) (fig. 25). Ainsi, malgré des traces d'occupation dès la fin du règne d'Auguste et l'existence d'une organisation de

26. S'il semble qu'il n'existe pas de bras vifs traversant la presqu'île à l'époque romaine, force est de constater que la compréhension de l'évolution de son occupation n'est pas simplifiée pour autant. En effet, jusqu'à la fin du $\mathbf{I}^{\mathrm{er}} \mathrm{s}$. après J.-C., celle-ci apparaît fortement liée à celle des composantes de la topographie, héritée du Premier Âge du Fer, sous l'action des flux thudaniens el de l'homme. D'autre part, on constate, de ce fait, de grandes disparités entre et à l'intérieur de plusieurs secteurs. Dans le secteur centre presqu'île, au nord de Bellecour, où se concentrent les occupations les plus anciennes, les premières constructions de l'îlot 24 , installées sur le haut de banc, ne s'étendent-elles en direction de la berge de la Saône que dans la seconde moitié du I ${ }^{\text {er }}$ s. après J.-C. Parmi les sites de Palais-Grillet ( $c f$. annexe I, $n^{\circ} 11$, p. 62), de la Bourse ( $c$. annexe I, $n^{\circ} 9$, p. 62) et de la République ( $c$. annexe $\mathrm{I}, \mathrm{n}^{\circ} 12$, p. 62-63), dont les premières fréquentations ou occupations augustéennes s'intercalent entre des dépôts de crues, seul le dernier d'entre eux est soumis au débordement du fleuve dans la seconde moitié du Ier ${ }^{\text {er }}$. après J.-C. Dans le secteur Bellecour, l'aménagement d'une levée de banc à la fin du $\mathrm{I}^{\mathrm{er}} \mathrm{s}$. après J.-C. sur le site de la station Bellecour ( $c f$. annexe I, $\mathrm{n}^{\text {os }} 17-17^{\text {bis }}-18$, p. 63) semble traduire un occupation relativement tardive de cette zone, alors que le site du Kiosque Bellecour porte les traces d'une occupation tibério-claudienne ( $c f$. annexe $\mathrm{I}, \mathrm{n}^{\circ} 19$, p. 63-64), et que celui des Facultés catholiques livre du mobilier de la fin du $\mathrm{I}^{\text {er }}$ s. après J.-C. ( $c$. annexe I, $\mathrm{n}^{\circ} 20$, p. 64) et celui de Widor les traces d'une occupation effective au milieu de ce siècle ( $c f$. annexe $\mathrm{I}, \mathrm{n}^{\circ} 22$, p. 64).

27. La description que donnent les témoignages du XVIII' s. de l'activité des chenaux au sud d'Ainay peut sans doute s'appliquer à ceux qui traversent la presqu'île à l'époque romaine : " toutes autres personnes passoient à pied secq a lexection [sic] de quelques temps de lannée que les eaux estoient grosses, dans lequel temps le Rosne y fesoit une petite brassière que l'on passoit facilement sur des pierres " (in: Audin, 1919, p. 66). En 1650, le Rhône recrée un lit principal à travers les jardins d'Ainay et transforme en île la partie sud de la presqu'île jusque-là rattachée. l'espace dans les décennies suivantes, la presqu'île demeure encore un milieu instable, non totalement assaini.

L'apparition sur presque tous les sites d'une occupation claudienne dont les structures obéissent à une même orientation nord-sud ${ }^{28}$, nonobstant quelques variations minimes et deux exceptions ${ }^{29}$, oblige à s'interroger sur l'existence ou non d'un projet d'urbanisme étendu à l'ensemble de la presqu'île. Un tel projet à la fin du règne d'Auguste, ou bien encore à l'époque claudienne comme cela avait été évoqué (Desbat, 1982; Desbat, Delaval, 1998), semble devoir être aujourd'hui écarté. En effet, durant tout le I ${ }^{\text {er }}$ s. après J.-C., l'occupation de la presqu'île paraît discontinue. La chronologie des vides sanitaires et des occupations, comme la nature de ces dernières, suggèrent des situations très différentes selon les lieux ${ }^{30}$.

À la fin du Ir $^{\text {er }}$. et au début du II ${ }^{\mathrm{e}}$ s. après J.-C., l'impression d'une occupation diffuse demeure même si la plupart des sites portent alors des vestiges d'habitat. Ce n'est qu'à la fin du I ${ }^{\text {er }}$ s. après J.-C. qu'une levée de terre consolidée par des amphores est élevée place Bellecour (cf. annexe $\mathrm{I}, \mathrm{n}^{\circ} 17, \mathrm{p} .63$ ) afin de protéger vraisemblablement des inondations la partie sud de la place. Les premières traces d'un habitat, sur le site des Célestins, n'apparaissent que dans le courant du II ${ }^{\mathrm{e}}$ s. après J.-C. Par ailleurs, seuls les sites de la rue Palais-Grillet ( $c f$. annexe I, $\mathrm{n}^{\circ} 11$, p. 62) et de la place de la Bourse ( $c f$. annexe $\mathrm{I}, \mathrm{n}^{\circ} 9$,

28. Cette orientation se retrouve notamment dans la rue mise au jour dernièrement sur le site de République-Bellecordière ( $c f$. annexe I, $\mathrm{n}^{\circ} 15$, p. 63 ).

29. Place des Célestins et place Chambonnet ( $c f$. annexe I, $\mathrm{n}^{\text {os }} 13$ et 16 , p. 63).

30. Les premières occupations diffèrent tant par leur date d'apparition que par leur nature. Ainsi, à Saint-Vincent, un secteur urbanisé est créé vers 15-10 avant J.-C., au plus tôt ( $c f$. annexe I, p. 60). Sur le site de l'îlot 24, les premières constructions sont élevées autour du changement de l'ère ( $c f$. annexe $\left.I, n^{\circ} 10, p .62\right)$; celles du Kiosque Bellecour sous Claude ( $c$. annexe I, $n^{\circ} 19$, p. 63-64). Si sur ces mêmes sites se développe un habitat, d'autres présentent au contraire des vestiges dont les matériaux, la densité et l'organisation correspondent plus vraisemblablement à une installation précaire ou bien à des zones de rejet. C'est le cas notamment des quelques fosses de la rue PalaisGrillet ( $c$. annexe $\mathrm{I}, \mathrm{n}^{\circ} 11$, p. 62), des traces anthropiques des places de la République et de Chambonnet ( $c f$. annexe I, $\mathrm{n}^{\text {os }} 12$ et 16, p. 62-63). Par ailleurs, les quelques rares dépôts d'amphores ou remblais augustéens ne laissent aucunement supposer qu'une vaste campagne d'assainissement ait été menée pendant cette période (îlot 24, cf. annexe I, $\mathrm{n}^{\circ}$ 10, p. 62 et Bellecour/Victor-Hugo, cf. annexe II, $\mathrm{n}^{\circ} 13$, p. 63). 


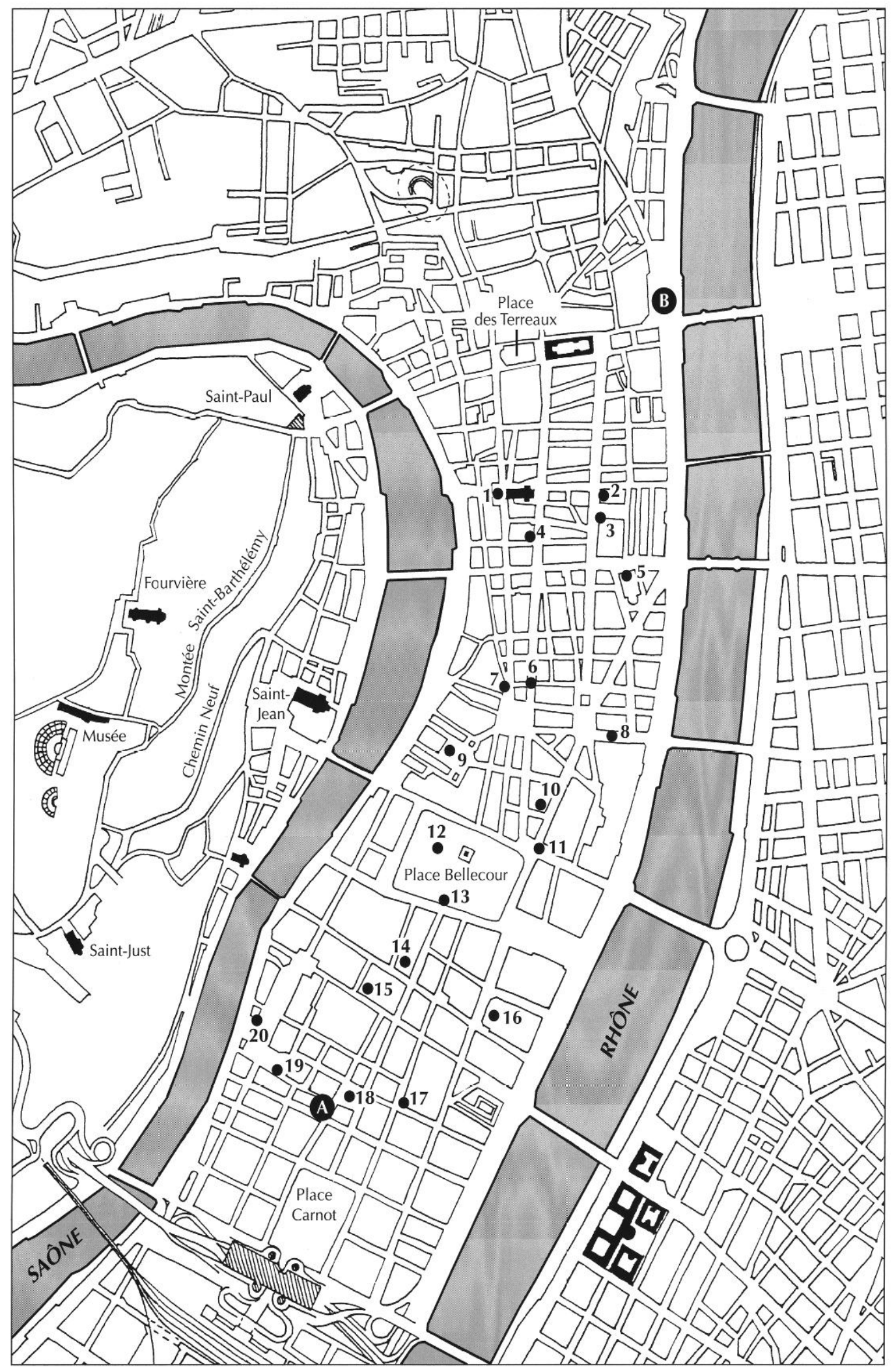

Fig. 25 - Carte des gisements d'amphores et des épaves (les $n^{o s}$ renvoient à ceux de l'annexe II) : $A$,épave rue Franklin; B, restes d'épaves sous le César, place Tolozan. 
p. 62) ont révélé des traces de constructions monumentales, malheureusement indéterminées, datées du début du II ${ }^{c}$ s. Sur la majorité des sites cependant, les vestiges semblent plutôt appartenir à des architectures modestes. On notera en particulier la construction d'un égout en bois à la fin du $\mathrm{I}^{\mathrm{er}} \mathrm{s}$. rue Bellecordière ( $c f$. annexe $\mathrm{I}, \mathrm{n}^{\circ} 15$, p. 63).

Ainsi, au $\mathrm{I}^{\text {er }}$ et au $\mathrm{II}^{\mathrm{e}}$ s. après J.-C., l'occupation de $\mathrm{l}$ a presqu'île semble se développer par extension à partir de noyaux primitifs en s'adaptant au milieu. Il est regrettable de ne pas pouvoir suivre cette évolution dans le détail au travers des nombreux vides sanitaires, malheureusement non datés, découverts au XIX ${ }^{\mathrm{e}}$ s. Il apparaît toutefois un contraste entre la partie centrale de la presqu'île et la partie méridionale, "l'île d'Ainay ", où l'on observe une occupation dense et plus riche, comme l'attestent les cinquante-trois mosaïques découvertes depuis le XVIII ${ }^{\text {s. }}{ }^{31}$ (Fabia, 1924, p. 53-114; Stern, 1967).

Il est très regrettable que les diverses opérations réalisées depuis plusieurs décennies n'aient jamais atteint la rive antique et révélé de digue ou de quai. Les traces d'enrochements retrouvés place Tolozan ( $c f$. annexe I, $\mathrm{n}^{\circ} 7$, p. 61) ou tout récemment rue Chambonnet ( $c f$. anncxc I, $\mathrm{n}^{\circ} 16$, p. 63) n'ćvoquent pas des travaux de grande ampleur. C'est également ce que suggère le site de l'îlot 24 (Jacquin et al., DFS, 1984) ( $c f$. annexe I, $\mathrm{n}^{\circ} 10$, p. 62), où l'habitat ne semble protégé que par un simple mur sur lequel les dépôts de crues s'appuient. Seule la fameuse digue signalée par Artaud paraît être un ouvrage important, mais sa nature exacte et surtout sa chronologie nous échappent encore (Artaud, 1846). Ce dernier nous signale également des pieux et enrochements ente la rue Sala et la rue Sainte-Hélène (id., ibid., p. 149). Les limites des berges nous sont tout de même connues en plusieurs points. Rue Dubois, une fouille réalisée en 1978 n'a révélé en effet aucune trace antique mais uniquement des pilotis modernes. Cela confirme que la rive antique était bien très en retrait de la rive actuelle et devait s'aligner sur la rue Mercière. L'extrémité de la presqu'île paraît bien fixée par l'épave signalée par A. Audin (1956, p. 133, fig. 6), mais il faudrait être certain qu'il s'agisse d'une épave antique. Par ailleurs, la découverte d'une soixantaine de monnaies

31. Pernetti signale dès le XVIII ${ }^{\mathrm{e}} \mathrm{s}$. «I a quantité de mosaïques qu'on a trouvé en faisant les fondations des maisons de la rue d'Enay. "
(Loriot, Rémy, p. 9), la plupart en or, à Perrache, dans la Saône, lors de la construction des pilcs du pont du chemin de fer, suggère soit l'existence d'une île au sud comme cela était le cas à l'époque moderne, soit le prolongement de la presqu'île au-delà d'Ainay.

\section{L'ÎLE SAINT-JEAN}

Les travaux récents ont confirmé l'existence du bras Marsaux et de l'île Saint-Jean décrite par Audin. Ils ont montré aussi que ce bras était le cours primitif de la Saône dont le colmatage débute dès le $\mathrm{I}^{\mathrm{cr}} \mathrm{s}$. et s'achève au III' s., à l'époque où le quartier connaît un début d'urbanisation. (Villedieu, 1990; Arlaud et al., 1994) (cf. annexe I, $\mathrm{n}^{\text {s }}$ 25-27, p. 64-65). Toutefois la configuration exacte de l'île Saint-Jean à l'époque romaine reste problématique. La présence d'un mur en travers de la Saône signalé par Artaud ${ }^{32}$ a suggéré l'existence d'une digue et l'avancée du rivage antique vers l'est, par rapport au rivage actuel. Bien qu'Artaud ait vu dans cette " muraille », plutôt les traces d'un aqueduc, l'hypothèse d'un rivage plus à l'est semblait confortée par le témoignage de Syméoni (rapporté par Spon et Montfalcon) qui signale " qu'il a vu et touché sous le pont de bois de Saint-Jean alors que la Saône était fort basse, des pavés et des débris de murailles » et par le témoignage de contemporains de Montfalcon ${ }^{33}$. Pourtant, les fouilles réalisées au chevet de l'église Sainte-Croix ont révélé l'existence d'un mur de digue ou d'une muraille du Bas-Empire, édifiée sur les berges de la Saône, en retrait à l'ouest du cours actuel! (Reynaud, 1998). Faut-il en conclure que le cours de la Saône a connu un déplacement important et que le lit du $I^{\mathrm{c}} \mathrm{s}$. est venu empiéter sur des terrains hors d'eau, occupés aux siècles précédents ?

32. Cf. Artaud, 1846 , p. 175 : " On distingue dans la Saône, lors de ses plus basses eaux, une muraille qui la coupe obliquement, à partir de la dernière pile du pont de l'archevêché à droite pour arriver à gauche vers la seconde arche du pont volant. Ce qu'on pourrait dire de plus raisonnable à l'égard de cette muraille c'est qu'elle a dû supporter des siphons ou cornets de plomb qui amenaient l'cau de la colline. »

33. Montfalcon (1847, p. 10, note 1) signale encore « que plusieurs personnes nous ont même assuré avoir également vu et touché à peu de profondeur en amont du pont Seguin un mur parallèle au courant de l'eau. Des constructions avaient donc existé sur ce point avant que la rivière y passât. " 
Il faut noter que les travaux récents n'ont concerné que la partie sud de l'île, ainsi la configuration de la partie nord nous échappe. Aucun élément nouveau n'est venu confirmer ou infirmer les hypothèses d'Artaud sur le secteur de la place du Change et sur l'existence d'un port à cet endroit (cf. infra, p. 56).

\section{LE DÉFILÉ DE PIERRE-SCIZE}

Les découvertes réalisées en 1965 et 1966 sur le quai Saint-Vincent ont montré l'existence d'installations artisanales, avec la présence d'ateliers de potiers qui s'installent dès la période augustéenne (ateliers de la Muette, de la place de la Butte, de la Manutention) (Desbat, Genin, Lasfargues éds, 1996 ; Desbat éd., 1997). À ces ateliers de potiers sont associées d'autres industries comme celles du verre et du bronze. Sur la rive droite, d'autres installations ont été repérées, en particulier les traces d'une activité céramique. Les fouilles du quai Arloing ( $c f$. annexe I, $n^{\circ} 33$, p. 65) ont confirmé la vocation artisanale des secteurs de berge avec l'existence d'ateliers métallurgiques ainsi que celle d'entrepôts.

Dans ce défilé se trouvait également la carrière d'où était extrait le micaschiste utilisé pendant plusieurs siècles dans les constructions. L'existence de cette carrière, voisine du rocher de Pierre-Scize, se lit encore dans la topographie actuelle. Un sondage réalisé en 1979, quai Pierre-Scize ( $c f$. annexe $\mathrm{I}, \mathrm{n}^{\circ} 30$, p. 65), a révélé de gros dépôts de schiste et des murs appartenant à des entrepôts ou des quais d'embarquement probablement liés à l'exploitation de cette carrière.

\section{LE QUARTIER DE VAISE}

Les travaux des géomorphologues ont confirmé qu'au début de l'époque romaine la plaine de Vaise est un milieu relativement stable. Sa rive gauche, construite au cours du Second Âge du Fer, est apparemment fixée. Son altitude, vers $164 \mathrm{~m}$ NGF pour ses parties les plus basses, et le régime particulier de la Saône font qu'elle est rarement atteinte par des débordements de crues.

Les découvertes récentes dans le quartier de Vaise ont montré l'importance, insoupçonnée jusqu'ici, de ce sec- teur de la ville. L'ancienneté de l'occupation qui remonte au Premier Âge du Fer ainsi que la présence de plusieurs gisements de La Tène démontrent l'existence d'une occupation préromaine liée sans doute à la voie commerciale que constitue la Saône dès les temps anciens. Sans que l'on puisse encore définir précisément le statut de cette occupation, il apparaît désormais que la colonie de Lugdunum est venue s'établir à proximité immédiate d'un site préromain important qui joue sans doute, dès le $\mathrm{II}^{\mathrm{c}} \mathrm{s}$. avant J.-C., le rôle d'emporium, comme l'a montré la découverte récente du site de la rue du Souvenir qui a livré en abondance des amphores gréco-italiques et Dressel 1 (Maza, 1998). La topographie de la plaine de Vaise, à l'abri des crues de la Saône, protégée par un cirque naturel formé par les plateaux de Fourvière et de la Duchère et le massif des Monts-d'Or, alimentée en eau par de nombreux ruisseaux, constituait à l'évidence un lieu plus hospitalier que la colline de Fourvière ou la presqu'île pour établir une occupation durable ${ }^{34}$. Mais c'est surtout le rôle de port naturel de Lyon que constitue le site qui paraît justifier, plus que tout autre raison, une installation à cet emplacement.

À l'époque romaine, le quartier est situé sans nul doute en dehors de la colonie, mais montre la présence d'une urbanisation structurée dès le début du $\mathrm{I}^{\text {er }} \mathbf{s}$. après J.-C. qui se développe de part et d'autre de la voie du Rhin et de l'océan. Les vestiges découverts place Valmy ( $c f$. annexe $\left.\mathrm{I}, \mathrm{n}^{\circ} 35, \mathrm{p} .65-66\right)$ et à Charavay ( $c f$. annexe $\mathrm{I}, \mathrm{n}^{\circ} 34$, p. 65) évoquent une occupation de type vicus. Les nombreuses traces d'artisanat relevées dans ce quartier témoignent d'une vocation artisanale et commerçante, semblable à celle des berges de la Saône, plus en aval. On rappellera la mise en évidence d'une production importante de laiton par cémentation sur le site de Valmy (Picon et al., 1995), ainsi que la présence d'installations hydrauliques, liées sans doute à un artisanat, pour l'instant mal défini (bassins et captage de Gorge de Loup) ( $c f$. annexe I, $\mathrm{n}^{\circ} 39, \mathrm{p} .66$ ), ainsi que la présence probable de moulins hydrauliques sur le site de la rue du Docteur-Horand ( $c$. annexe I, $\mathrm{n}^{\circ} 38$, p. 66).

34. Le caractère inhospitalier du site de Lyon a été maintes fois souligné. La première critique est due à Sénèque dans l'Apocolokincose, mais on trouvera d'autres arguments chez. Drinkwater, 1975 : "Lugdunum, natural capital of Gaule ». 


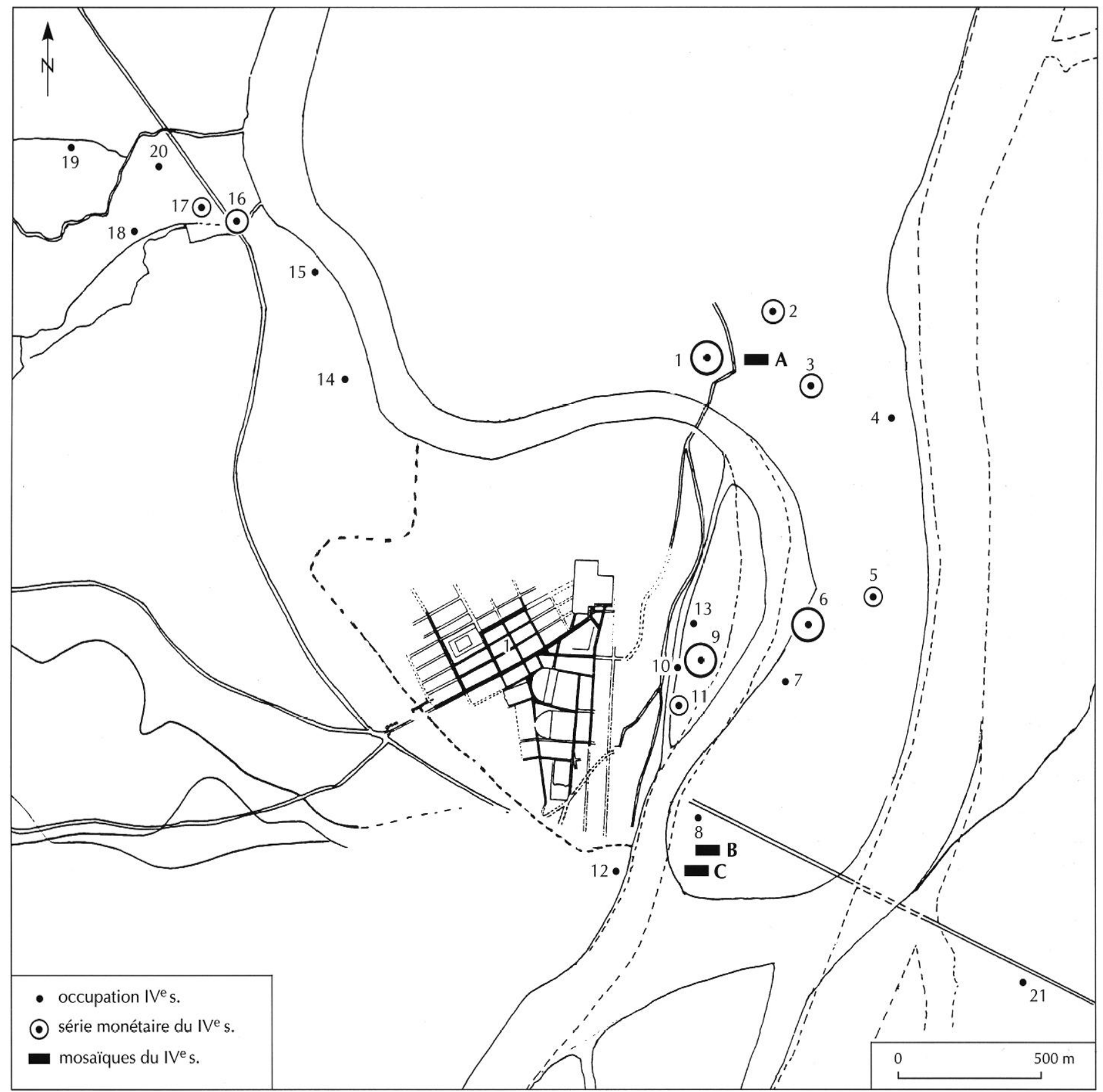

Fig. 26 - Occupations ou traces d'occupation du Bas-Empire ( $I V^{p}$ et $\left.V^{e} s.\right)$.

Sites (les $n^{o s}$ renvoient à ceux de l'annexe $\left.I\right): 1$, Saint-Vincent (secteurs III et I), occupation jusqu'au V's. après J.-C. sur le secteur III $\left(n^{\circ} 1\right)$; 2, îlot Vieille-Monnaie $\left(n^{\circ} 3\right) ; 3$, Terreaux $\left(n^{\circ} 4\right) ; 4$, Bourse $\left(n^{\circ} 9\right) ; 5$, Palais-Crillet $\left(n^{\circ} 11\right) ; 6$, îlot 24, encore occupé au Haut Mojen Âge $\left(n^{\circ} 10\right) ; 7$, Célestins, site encore occupé entre les VII et IX ${ }^{e}$ s. après J.-C. $\left(n^{\circ} 13\right) ; 8$, Sainte-Hélène $\left(n^{\circ} 21\right)$; 9 , station Saint-Jean, site occupé aux Ve et $V^{e}$ s. après J.-C. mais également au cours du Haut Moyen Âge $\left(n^{\circ} 26\right) ; 10$, funiculaire Saint-Jean, site occupé après le IV $V^{e}$ s. après J.-C. $\left(n^{\circ} 27\right) ; 11$, Clément-V, site encore occupé aux Ve-VTe s. après J.-C. ( $\left.n^{\circ} 25\right) ; 12$, Quarantaine $\left(n^{\circ} 24\right) ; 13$, maison du Chamarier, site encore occupé au Haut Moyen Âge $\left(n^{\circ} 28\right) ; 14, L^{\prime}$ Observance $\left(n^{\circ} 31\right) ; 15$, Arloing, occupation et nécropole $\left(n^{\circ} 33\right) ; 16$, Charavay $\left(n^{\circ} 34\right)$; 17 , nécropole de Valmy $\left(n^{\circ} 35\right) ; 18$, voie Saint-Pierre, site encore occupé au Haut Moyen Âge $\left(n^{\circ} 41\right) ; 19$, Horand $\left(n^{\circ} 38\right) ; 20$, Les Blanchisseries $\left(n^{\circ} 37\right) ; 21$, Cheurier $\left(n^{\circ} 42\right)$.

Mosaïques du IV s. après J.-C. : A, 3 place Sathonay (seconde moitié du III s. ou $I^{e}$ s. après J.-C.) ; B, 3 rue Jarente (IVe-Ve s. après J.-C.) ; $C, 4$ rue Jarente (tardive). 


\section{LA TRAVERSÉE DES FLEUVES}

L'arrivée de la voie d'Italie sur la rive gauche, repérée récemment encore rue du Père-Chevrier ( $c f$. annexe I, $\mathrm{n}^{\circ} 42$, p. 66) et matérialisée par les mausolées trouvés au XIX $^{e}$ s., suppose une traversée du Rhône dans l'axe de la place Gailleton. On a inféré l'existence d'un pont sur le Rhône du récit de l'assassinat de l'empereur Gratien en 383 (Artaud, 1846, p. 120 ; Steyert, 1895, p. 481), mais ni la nature exacte de ce pont ni la date de sa création ne nous sont connues. Il paraît exclu qu'un pont de pierre ait existé à cet emplacement et il faut envisager plutôt un pont de bateaux comme à Arles ou encore un bac comme il en existait encore au XIX ${ }^{e}$ s. La découverte dans le lit du Rhône de nombreux blocs antiques et de pieux au $\mathrm{XIX}^{\mathrm{e}}$ s. (Gobin, 1872) confirme l'avancée vers l'ouest de la rive gauche à l'époque antique (fig. 26). Il est difficile de dire si les blocs sont ceux de mausolées déplacés par les crues du Rhône, ou s'il s'agit plutôt de blocs réutilisés à une époque plus récente pour stabiliser la berge, comme le pensaient Allmer et Dissard (1889). Les pieux eux-mêmes pourraient correspondre dans la première hypothèse à un appontement ou à la fondation de la voie antique établie sur des terrains alluvionnaires peu stables. Quoi qu'il en soit, le passage du Rhône dans l'axe de la rue Sainte-Hélène semble bien attesté, sans que l'on puisse toutefois dater avec précision son installation. $\mathrm{Ya}$ t-il eu antérieurement une traversée du Rhône plus au nord ? Seule la découverte de tombes datées de la première moitié du $\mathrm{I}^{\mathrm{er}} \mathrm{s}$. pourrait nous fournir une réponse.

Un deuxième passage devait exister au débouché de la rue Sainte-Hélène pour la traversée de la Saône. Cette hypothèse défendue par Audin semble plausible, même si aucun vestige n'est venu la confirmer, et l'on ignore de même si la traversée se faisait par bac ou par un pont.

On peut supposer un pont sur la Saône, communication entre la colonie et le sanctuaire fédéral. L'ancienneté du quartier Saint-Vincent, mais aussi la présence des deux églises Saint-Paul et Saint-Vincent, de part et d'autre de la Saône, suggèrent l'existence d'un pont à l'emplacement de la passerelle Saint-Vincent. Une autre possibilité serait de placer ce pont un peu plus en aval dans l'axe de l'ancienne place de la Douane et de la place de la Platière où $\mathrm{B}$. Gauthiez propose de situer le pont médiéval construit au XI ${ }^{\mathrm{c}} \mathrm{s}$. sur la Saône (Gauthiez, 1994, p. 14).

\section{LES PORTS DES NAUTES}

La question des ports n'a pas vraiment progressé depuis les hypothèses anciennes dont la synthèse a été présentée en 1982, par l'un de nous (Desbat, 1982). Sans aller jusqu'à imaginer un port de type maritime flanqué de phares comme l'avait restitué Chenavard, la plupart des auteurs ont supposé l'existence de trois ports au moins sur les berges actuelles de la Saône (Artaud, 1846 ; Steyert, 1895; Audin, 1964) : le premier à la hauteur de Perrache ; le deuxième à la hauteur de Saint-Georges ; le troisième à la hauteur de Saint-Paul. À ces trois ports situés sur la rive droite de la Saône, Steyert (1895) ajoutait le port des vinarii installé dans les Canabées en face de celui de Saint-Georges.

L'essentiel des données concernant les ports supposés est tiré d'Artaud, mais aucune découverte récente n'est venue confirmer la réalité de leur existence. Leur localisation s'appuie sur la découverte de nombreux sceaux ou tessères de plomb recueillis dans la Saône près du pont du Change et de la passerelle Saint-Vincent (Turcan, 1987). Toutefois, des découvertes semblables ont été effectuées sur un parcours de plus de $2 \mathrm{~km}$ lors de la construction des quais, à partir de 1858 , aussi bien sur la rive droite que sur la rive gauche (Dissard, 1905). Cela tendrait à valider l'hypothèse de "ports " au sens de débarcadères, disposés de manière continue le long des rives, comme nous le montrent les plans de Lyon au $\mathrm{XVIII}^{\mathrm{e}} \mathrm{s}$., où l'on dénombre pas moins de dix-neuf ports échelonnés sur les rives du Rhône et de la Saône ${ }^{35}$.

Malgré les nombreuses opérations réalisées dans la presqu'île, il faut constater que les aménagements de berge nous ont échappé jusqu'ici. Aucune fouille récente n'a révélé de quai comme pour le port de Londres (Milne, 1985 ; Miller et al., 1986), ni même des horrea de l'ampleur de ceux connus à Vienne (Helly-Lebot, 1989). On peut tout de même signaler sur les berges de la Saône à Vaise (rive droite), quai Arloing (Tranoy, 1995), et en aval, quai Pierre-Scize ( $c f$. annexe I, $\mathrm{n}^{\circ} 30$, p. 65 ), l'exis-

35. Sur le plan de Séraucourt de 1740 , on trouve d'aval en amont, sur la Saône en rive droite : le port Sablet, le port Saint-Jean, le port de Roanne, le port Dauphin, le port de Saint-Paul, le port de l'Arche, le port de la Roche, le port de l'Observance ; sur la Saône en rive gauche: le port du Roy, le port du Temple, le port Saint-Antoine, le port de Chalamont, le port de la Feuillée, le port Saint-Benoît, le port Neuville ; sur le Rhône : le port de l'Hôpital, le port des Cordeliers, le port des Jésuites, le port de Saint-Clair. 
tence de grands murs parallèles qui pourraient appartenir à des entrepôts, de même qu'à Saint-Vincent, sur la rive gauche ( $c f$. annexe $\left.\mathrm{I}, \mathrm{n}^{\circ} \mathrm{l}, \mathrm{p} .60\right)$ ). On peut encore mentionner l'existence de docks à dolia découverts quai des Étroits en 1964 (Audin, 1964) ou encore des dolia aperçus lors de la construction du parking de Bellecour en 1966, sur le côté occidental de la place ${ }^{36}$.

\section{LES QUARTIERS FLUVIAUX : LA VILLE NOUVELLE DE LUGDUNUM?}

De même que Morand et Perrache, au XVIII ${ }^{c}$ s., ont cherché à agrandir la ville en gagnant des terrains sur lcs fleuves ou les zones soumises aux crues, il semble bien que l'occupation de la presqu'île ou du quartier SaintJean corresponde à une extension tardive de la ville. Ces secteurs semblent constituer, en effet, des quartiers neufs comparés à ceux de Fourvière qui forment la vieille ville, où l'habitat est ancien et les possibilités de créations limitées, notamment à cause de la topographie accidentée. On peut opposer les sites de la rue des Farges ou du Verbe-Incarné, où se maintient un habitat qui conserve les limites et les plans créés au ${ }^{\mathrm{er}} \mathrm{s}$., aux riches maisons à mosaiques qui se développent en particulier dans le quartier d'Ainay. La présence de ces riches domus semble indiquer un quartier riche, avec une fonction à la fois commerçante, avec le siège des corporations de negotiatores, et résidentielle, avec les demeures de ces riches commerçants. À bien des égards, ce quartier des Canabées s'apparente à celui de Saint-Romain-en-Gal ou aux quartiers sud de Vienne qui regroupent de grandes et riches demeures et des installations artisanales ou commerciales.

La principale interrogation porte sur le statut de ces quartiers, logiquement hors du pomerium, à moins de supposer une extension de la ville à partir de Claude, comme pourrait le suggérer la nouvelle titulature de Lyon "Colonia Copia Claudia Augusta Lugdunum " (Desbat, Delaval, 1998) et la création du compendium Lyon-Vienne.

Le développement des quartiers fluviaux aux $\mathrm{II}^{\mathrm{e}}$ et $\mathrm{III}^{\mathrm{e}}$ s. constitue l'amorce d'un déplacement du centre de gravité de la ville qui sera effectif au Bas-Empire et au

36. Cctte découverte n'est connue que par le témoignage de M. Picun présent sur place au moment de leur misc au jour.
Moyen Âge, avec l'abandon de la ville haute. L'abandon de la colline de Fourvière engagé dès le III' $s$. semble définitif au IV $\mathrm{V}^{\mathrm{re}} \mathrm{s}$., même si l'on peut considérer que certains monuments continuent peut-être à être utilisés, comme le théâtre ou l'odéon ${ }^{37}$.

Les troubles qui interviennent en Gaule durant le III ${ }^{\mathrm{s}} \mathrm{s}$. ont sans doute eu une incidence sur l'évolution des quartiers fluviaux. On ignore quelles ont été les conséquences du sac de la ville par Aurélien en 273, mais il est certain que des événements dont on mesure mal l'ampleur ont affecté la presqu'île à la fin du III's. La présence de dépotoirs de cette période dans les égouts de la place Louis-Pradel ( $c f$. annexe $\mathrm{I}, \mathrm{n}^{\circ} 6$, p. 60 ) ou de la rue Bcllccordic̀re ( $c f$. annexe $\mathrm{I}, \mathrm{n}^{\circ} 15$, p. 63 ) traduit l'abandon d'une partie des infrastructures, à l'époque où l'on érige précisément sur le site de l'îlot Vieille-Monnaie ( $c f$. annexe $\mathrm{I}, \mathrm{n}^{\circ} 3, \mathrm{p} .60$ ) un mur qui pourrait être un élément de l'enceinte réduite.

\section{LA VILLE DU BAS-EMPIRE}

L'existence d'une agglomération organisée autour de deux pôles sur les berges de la Saône remonte sans doute à l'époque romaine. Il est possible en effet que les bourgs fortifiés connus au Moyen Âge autour de Saint-Jean et de Saint-Pierre reprennent en partie des tracés romains et que le noyau 1B de la ville médiévale proposé par B. Gauthiez (1994, fig. 4 et 21) corresponde en partie aux contours de la ville du Bas-Empire. Sur la rive droite, le mur édifié au IV $\mathbf{s}$. au chevet de l'église Sainte-Croix (Reynaud et al., 1978; Reynaud, 1998) constitue sans doute un élément d'une enceinte, soit limitée au cloître de Saint-Jean, soit plus vaste. Bien que J.-F. Reynaud ait abandonné récemment l'idée d'une enceinte de SaintPaul à Saint-Georges, faute de preuve (Reynaud, 1998, p. 187), une prolongation du mur jusqu'à Saint-Georges n'est pas à exclure ${ }^{38}$.

37. En effet, les fouilles du théâtre ont livré des monnaies des IVe et $V^{e} s$., la monnaie la plus récente étant de Julien l'Apostat (Wuilleumier, 1951).

38. En 1840, lors de la construction du quai Fulchiron, entre le pont Bonaparte et l'ancien pont d'Ainay, la tranchée rencontra des lignes de pilotis (Comarmond, 1840, p. 66). La date de ces pilotis reste indéterminée, mais la présence de blocs d'architecture romains entre les pieux suggère qu'il s'agit peut-être de la fondation du prolongement du mur digue ou rempart découvert à Saint-Jean. 


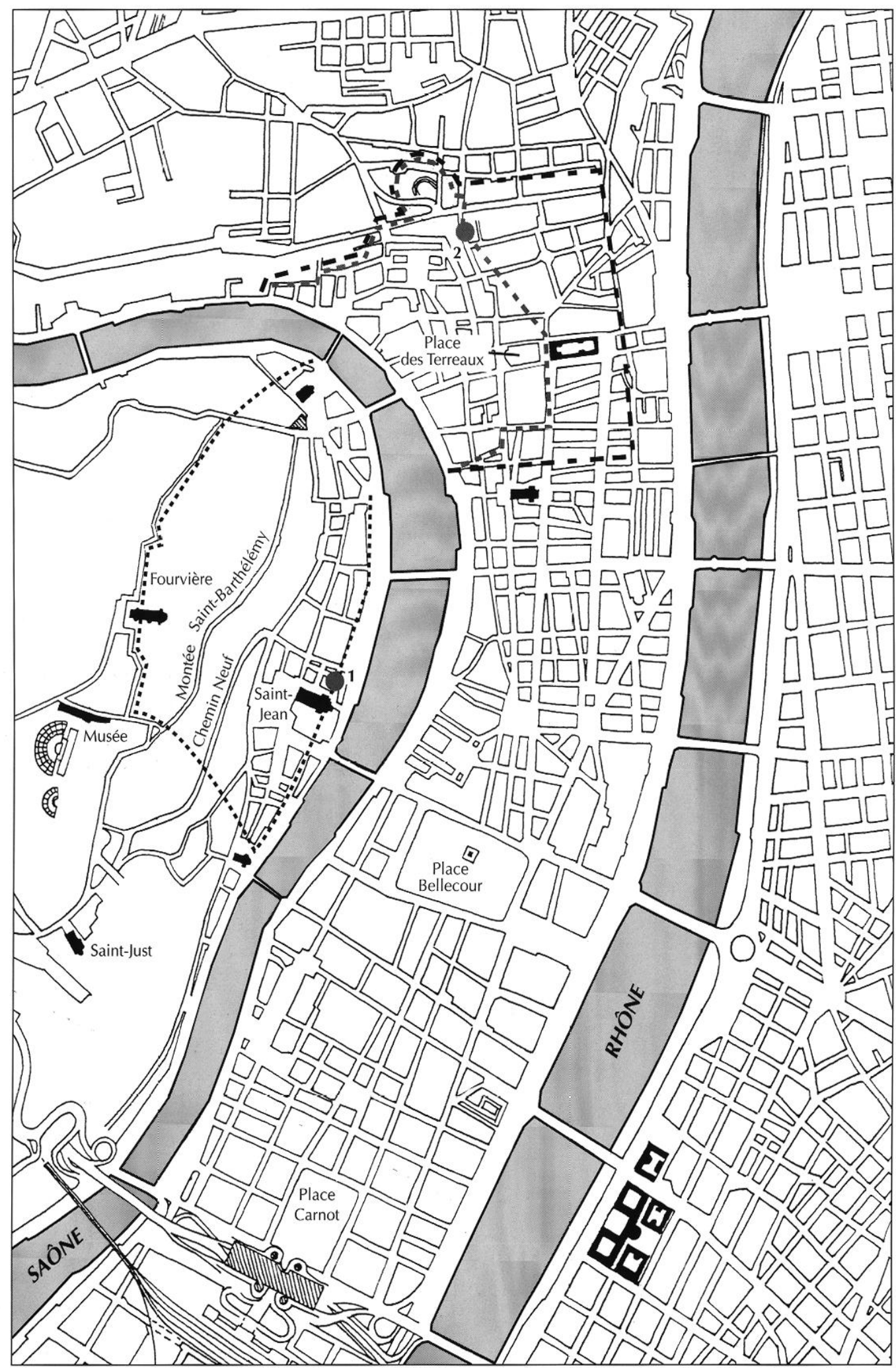

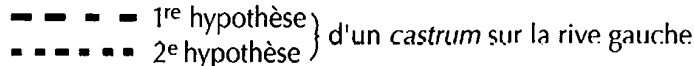

......... tracé supposé de l'enceinte sur la rive droite de la Saône
Fig. 27 - Hypothèses des tracés des enceintes réduites du Bas-Empire:

1, mur du groupe épiscopal;

2, mur de l'̀̂lot Vieille-Monnaie. 
L'abandon de la ville haute et la topographie de la presqu'île rendent également très probable l'existence d'un castrum au Bas-Empire, sur la rive gauche, entre Rhône et Saône. Le témoignage de Grégoire de Tours sur la destruction d'une partie des remparts de la ville en 580 , lors d'une crue du Rhône, va dans ce sens ${ }^{39}$. La fameuse digue d'Artaud sous la rue Mercière (Artaud, 1846) pourrait être un élément de ce système défensif, à la fois rempart contre les eaux et contre les menaces extérieures, comme celui de Saint-Jean. Faute de données chronologiques sur la «digue » d'Artaud, il est impossible de répondre avec certitude, mais faire de ce mur un élément de l'enceinte conduirait à lui donner une longueur importante.

Plusieurs autres tracés peuvent être envisagés pour ce castrum : soit une enceinte limitée prenant appui sur l'amphithéâtre, et circonscrite au quartier Saint-Vincent, soit une enceinte plus large englobant Saint-Nizier voire le quartier jusqu'au Rhône (fig. 27).

L'absence jusqu'ici de traces d'occupation tardive à l'est de la place des Terreaux (Hôtel de Ville, place LouisPradel, Opéra, $c f$. annexe I, n` ${ }^{\circ-8}$, p. 60-62) nous ferait plutôt privilégier l'hypothèse d'un bourg limité au quartier Saint-Vincent et à la Platière ${ }^{40}$.

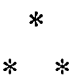

39. Grégoire de Tours, Histoire des Francs: "En même temps le Rhône et la Saône sortant de leurs lits causèrent de graves dommages aux populations voisines et renversèrent une partie des murailles de la ville de Lyon " (cap. IV, § 57).

40. Cette hypothèse n'exclut pas pour autant l'existence d'occupations extra-muros. Les traces de fréquentations ou d'occupations du IV ${ }^{\mathrm{e}} \mathrm{s}$. ont été décelées place des Terreaux, rue Palais-Grillet ou encore place des Célestins.
Les données archéologiques récentes comme les observations géomorphologiques sont aujourd'hui suffisamment convergentes pour établir que les premières occupations s'installent sur des terrasses à l'abri des crues, autour de la cote 166, que ce soit à Saint-Vincent ou à Vaise. La plaine d'inondation dont les géomorphologues nous restituent l'image à l'Âge du Fer n'est aménagée que tardivement. Les nombreux points de découverte sur la presqu'île confirment l'aménagement récent de ce secteur par rapport à la colline, en contradiction avec l'idée souvent répandue chez les auteurs anciens du port cosmopolite, la " marine ", accueillant Grecs et Asiates à côté de l'oppidum celtique ayant précédé la ville romaine (Lenthéric, 1892).

L'ancienneté des occupations rencontrées à Vaise et notamment l'importance de l'occupation laténienne, insoupçonnée jusqu'à une date récente, montrent que le site du confluent, au sens large, a bel et bien constitué un pôle d'attraction important, et fut un élément clé de la genèse de la ville, contrairement à une opinion souvent défendue ${ }^{41}$.

Parmi les questions qui demeurent figure en premier la question des limites du pagus de Condate et des Canabées. L'existence d'un bras du Rhône fournissait une solution commode par le passé mais aujourd'hui la question reste entière.

41. Contrairement à l'avis de Y. Burnand (1977, p. 279) qui constatait : " le manque d'attraction exercéc par les cours d'eau rhodaniens, en particulier sur la fixation des agglomérations ante romaines ». Cf. également : "Dans le passé pré-romain c'est en dehors du fleuve que le peuplement va chercher ses centres de commandement ou ses foyers de rayonnement " (Faucher, 1968). L'auteur admet que la seule exception est Vienne! mais on peut lui opposer Lyon, Mâcon, Chalon-surSaône, etc. 


\section{ANNEXE I}

\section{OPÉRATIONS ARCHÉOLOGIQUES RÉALISÉES DEPUIS 1978 DANS LA PRESQU'ÎLE ET LES QUARTIERS FLUVIAUX (fig. 24 et tabl. I)}

\section{Avertissement}

Les interprétations et les datations sont données sous réserves de modifications qui pourraient être apportées par les auteurs au moment de la publication des résultats.

\section{Abréviations}

CS : campagne de sondages.

FSU : fouille de sauvetage urgent ou suivi de terrassement.

FSP : fouille de sauvetage programmé.

\section{LA PRESQU'ÎLE}

1. Saint-Vincent (ZAC du quartier, rues Bouteille, F.-Rey, Tourret et de la Vieille) : $6000 \mathrm{~m}^{2}$ répartis principalement en trois secteurs; FSU et FSP (Lascoux et al., DFS, 1986, 1987, 1989, 1994).

Période 1 (tous secteurs) : niveaux alluviaux recouverts par des colluvions (sup. entre 166,50 et $168 \mathrm{~m} \mathrm{NGF).} \mathrm{Période} 2$ (secteur III) : traces ténues d'une occupation du Bronze final. Période 3 (secteur III) : traces ténues d'une occupation de la fin du Premier Âge du Fer. Période 4 (secteurs II et III) : atclier de potier du milieu du I ${ }^{\mathrm{er}}$ s. av. J.-C. Période 5 (secteur II) : vestiges très lacunaires d'une construction isolée, occupée entre 30 et $15 / 10$ av. J.-C. Période 6 : urbanisation générale du site vers $15 / 10$ av. J.-C. au plus tôt ; phase 1, fin du I ${ }^{\text {er }}$ s. av. J.-C., entrepôts et habitats ; phase 2 , courant I ${ }^{\mathrm{er}}$ s. apr. J.-C., création d'un habitat et d'un espace ouvert public ou privé à l'emplacement des entrepôts ; nouveaux habitats associés ou non à de l'artisanat. Période 7 : début du II ${ }^{\mathrm{c}}$ s. apr. J.-C., apparition d'une grande demeure occupant au moins deux parcelles antérieures sur le secteur I; construction de thermes et maintien de l'espace public ou privé sur les secteurs II et III. Période 8: au III et IVes. apr. J.-C. maintien des thermes et de la grande demeure de la période précédente; réapparition d'un artisanat sur le secteur I. Période 9 : abandon, vraisemblablement progressif, du site entre le IV et le VIc s. apr. J.-C.

2. Martinière (au niveau du 3-5 rue de la) : $2 \mathrm{~m}^{2}$; CS (Desbat, Walker, 1981).

Séquence gallo-romaine entre 167,70 et $169,30 \mathrm{~m}$ NGF. Les niveaux les plus anciens apparaissent au plus tôt aux alentours de 30 apr. J.-C. ; les plus récents témoignent d'un incendie entre l'extrême fin du III' et la fin du IV ${ }^{e}$ s. apr. J.-C.

3. Îlot Vieille-Monnaie I (73-89 montée de la Grande Côte) ; partie sud du site $500 \mathrm{~m}^{2}$; FSP (Jacquin et al., DFS, 1985a et b).

Période 1 : dans la dernière décennie avant l'ère, construction d'un portique, d'une grande salle et de trois petites pièces, partie d'un habitat plus vaste. Période 2 : entre 20-50 apr. J.-C. et la fin du II' s. apr. J.-C., extensions progressives des constructions aux dépens du portique. Période 3 : fin du II' s. apr. J.-C., nouvelle campagne de construction. Période 4 : troisième quart du III ${ }^{e}$ s. apr. J.-C., construction d'un mur large de $1,80 \mathrm{~m}$, peutêtre le tronçon d'une enceinte réduite. Période 5 : milieu du $\mathrm{IV}^{\mathrm{c}} \mathrm{s}$. apr. J.-C., traces d'une fréquentation sporadique à l'intérieur de l'habitat, puis abandon de ce dernier au début du IV ${ }^{\mathrm{e}} \mathrm{s}$. apr. J.-C.

$3^{\text {bis }}$. Îlot Vieille-Monnaie II (73-89 montée de la Grande Côte) ; partie nord du site $500 \mathrm{~m}^{2}$; CS (Plassot et al., DFS, 1991a).

Au sud du site, continuation de l'habitat du site précédent (sols entre 184,84 et 185,95 m NGF) ; au nord, énorme maçonneric orientée est-ouest, d'une largeur minimale de $15 \mathrm{~m}$ et d'une hauteur minimale de $8,50 \mathrm{~m}$ (sup. 193,34 m NGF) ; aucun élément de datation.

4. Terreaux (parking des, place des); à l'emplacement du fossé de la fortification médiévale : $3500 \mathrm{~m}^{2}$ dont $700 \mathrm{~m}^{2}$ en quatre zones concernant l'Antiquité ; FSP (Arlaud et al., DFS, 1994).

Période 1 : substrat caillouteux d'origine rhodanienne (sup. $163,40 \mathrm{~m} \mathrm{NGF}$ ), recouvert par des épisodes d'alluvionnement du Rhône et de la Saône. Période 2 : séquence à dominante colluviale (sup. 165,20 m NGF) renfermant des traces anthropiques non datées. Période 3: traces d'occupation du II' ${ }^{\mathrm{s}}$ s. apr. J.-C. (sols composés de débris de calcaire, niveaux de gneiss, etc.), suivies, dans le courant du III ${ }^{\mathrm{e}}$ s. apr. J.-C., d'une phase d'alluvionnement. Période 4 : construction du $\mathrm{IV}^{\mathrm{c}} \mathrm{s}$. apr. J.-C., détruite vers la fin $d u V^{e}$ s. apr. J.-C. ; à l'intérieur de cette période, sont regroupés plusieurs autres ensembles fragmentaires, dont deux pièces disposées en T de la fin de l'Antiquité tardive, des fragments de sol, de mur et des trous de poteaux non datés (entre 166 et 166,50 m NGF). Période 5 : trois sépultures non datées.

5. Hôtel de Ville (annexe de 1', place Louis-Pradel) : $100 \mathrm{~m}^{2}$; CS (Mandy et al., DFS, 1981).

Dans la partie nord, vestiges fragmentaires de sols et de deux maçonneries parallèles; dalles de voies extraites des puits de fondation ; mobilier atypique hormis celui de la tranchée de construction d'un des murs ( ${ }^{\mathrm{er}} \mathrm{s}$. apr. J.-C.) ; au sud, sol non daté à $167,90 \mathrm{~m}$ NGF.

6. Louis-Pradel (parking, place) : estimée $1200 \mathrm{~m}^{2}$; FSU (Helly, DFS, 1979).

Plusieurs ensembles incomplets, composés de murs et de fragments de sols (hypocauste, terrazzo, vestiges d'une mosaique), datés entre le I ${ }^{\mathrm{er}}$ et le III ${ }^{\mathrm{e}}$ s. apr. J.-C. ; l'un des sols reposait sur une séquence alluvionnaire contenant du mobilier du I ${ }^{\mathrm{cr}}$ s. apr. J.-C. ; un égout, orienté nord-sud, contenait un mobilier du $\mathrm{III}^{\mathrm{e}}$ s. apr. J.-C. 
Tabl. I - Alitudes des différentes phases d'occupation des sites.

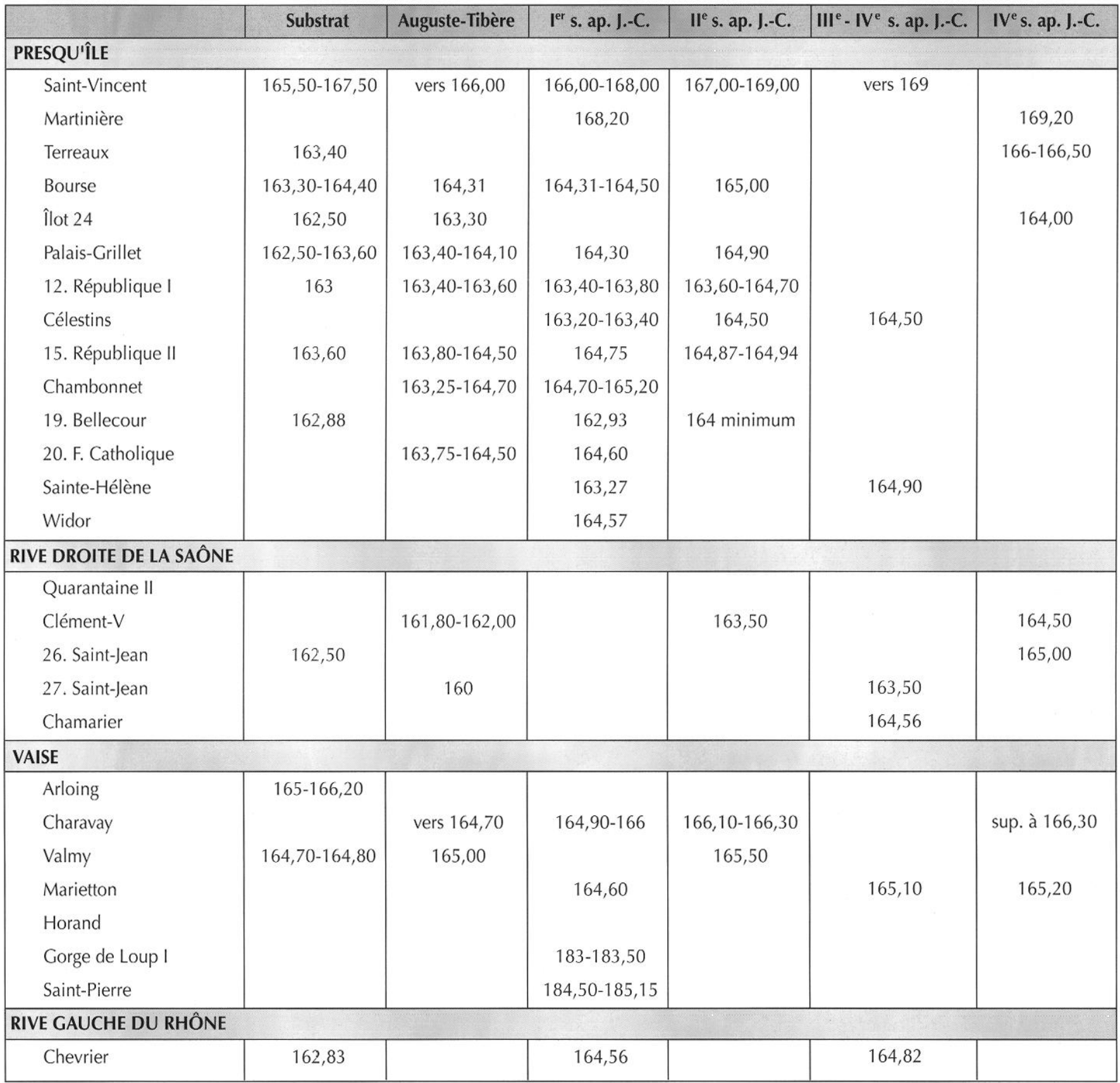

7. Le César (22-23 place Tolozan) : $800 \mathrm{~m}^{2}$; FSP (Becker, Rieth, 1995).

Deux murs, un caniveau gallo-romains et une démolition considérée, peut-être à tort, comme les traces d'un quai ou d'un mur de soutènement; vestiges d'une épave reposant en partie sur un cailloutis rhodanien, ct un enrochement de gros blocs de granite disposé perpendiculairement à la berge ; datation ${ }^{14} \mathrm{C}$ sur deux bois de l'épave, intervalle en âge calibré : 8 av. J.-C./230 apr. J.-C. et 66 apr. J.-C./234 apr. J.-C. (laboratoire de radiocarbone lyon I); datation dendrochronologique sur quatre bois de la même épave : vers 30 av. J.-C., pas antérieure à 20 av. et vers 35 av. J.-C. ; ARC 90/R804D (Archéolabs).

8. Opéra (place de la Comédie) : $310 \mathrm{~m}^{2}$; FSU (Raynaud $e t$ al., DFS, 1990).

À l'emplacement du fossé de la fortification médiévale du XII ${ }^{\mathrm{e}}$ s. apr. J.-C. : restes d'un puits daté par ${ }^{14} \mathrm{C}$ de la fin du $\mathrm{I}^{\mathrm{cr}} \mathrm{s}$. apr. J.-C., deux murs et des niveaux de sol dont un, de la fin du 
III s. apr. J.-C., scellé par un épisode de colluvionnement culminant à 163,70 m NGF.

9. Bourse (place de la) : $1700 \mathrm{~m}^{2}$; FS (Arlaud et al., DFS, 1990a).

Période 1 : cailloutis rhodanien (sup. $163,30 \mathrm{~m}, 164,42 \mathrm{~m}$ NGF), surmonté de dépôts de crues issus de débordements du Rhône. Période 2 : un pilier (sup. 162,57 m NGF) récupéré ou détruit par de fortes crues avant une inondation générale du site. Période 3 : dans la partie sud-est, un niveau de sol avec du mobilier de la seconde moitić du règne d'Auguste, scellé par un dépôt de débordement du Rhône. Période 4 : dans le même secteur, un foyer et du mobilier de la fin du règne d'Auguste, clos par un nouvel épisode d'inondation. Période 5 : au début du règne de Tibère, extension de l'occupation dans les partics est, centrale et nord-ouest du site, représentée par des suls, des trous de poteaux et des fosses; un retour de crue, recouvrant presque tout le site (sup. 164,31 m NGF), scelle ces vestiges. Période 6 : deux piliers et deux murs dans la partie sudest du site ; les maçonneries, récupérées sous le règne de Tibère ou au début de celui de Claude, sont fermées par un dernier épisode de crue. Période $7:$ milieu du ${ }^{\text {er }}$ s. apr. J.-C. dans le même secteur, un sol de terre battue et un foyer. Période 8 : phase 1 , à la fin du règne de Claude ou au début de celui de Néron, un apport de graviers précède l'apparition de la façade d'un nouveau bâtiment à l'est ; phase 2, apparition de nouvelles constructions dans la partie centrale et occidentale; phase 3 , sous les Flaviens, destruction partielle ou totale de l'cnsemble des bâtiments par un incendie. Période 9 : récupération, nivellement et exhaussement du site de 0,30 à $0,40 \mathrm{~m}$ (sup. vers $165 \mathrm{~m}$ NGF) ; dans la partic ouest, la plus densément construite, un bâtiment aux fondations puissantes est élevé au début du $\mathrm{II}^{\mathrm{e}} \mathbf{s}$. apr. J.-C., puis détruit à la fin de ce siècle ou au début du suivant. Période 10: des trous de poteaux et de rares murs de galets liés à la terre témoignent d'une occupation au IV s. apr. J.-C. ; après cette date, le site est apparemment déserté.

10. Îlot 24 (rue de la Monnaie) : $450 \mathrm{~m}^{2}$; FSP (Jacquin et al., DFS, 1984 ; Jacquin et al., 1993).

Secteur $I$ : deux pièces et un bassin, partie d'un bâtiment plus vaste élevé au sommet d'un banc caillouteux, autour du changement d'ère.

Secteur II-Période 1 : banc caillouteux construit par le Rhône, recouvert de sédiments fins déposés par des crues de la Saône (sup. moy. 162,50 m NGF). Période 2 : construction d'un mur, peut-être une digue destinée à protéger le site des eaux, suivie d'un apport de remblai (sup. moy. 163,30 m NGF); puis construction à l'est de ce mur d'un habitat occupé entre 30 et 60 apr. J.-C. Période 3 : extension jusqu'au mur-digue et restructuration de l'habitat; à l'ouest, dans la partie centrale du site, apparaît un espace ouvert doté d'un réseau de canalisations probablement en bois; au sud-ouest, des piliers et deux murs prouvent que des constructions se développent, en direction de la Saône, à une date indéterminée. Période 4 : reconstruction à la fin du II ${ }^{\mathrm{e}}$ s. apr. J.-C. modifiant l'organisation interne de l'ha- bitat. Période 5 : nouvelles modifications, puis abandon de l'habitat au milieu du III ${ }^{\mathrm{e}} \mathrm{s}$. apr. J.-C. Période 6 : fragments de sols en terre battue témoignant d'une occupation du site au IVe $s$. apr. J.-C. (sup. vers $164 \mathrm{~m} \mathrm{NGF),} \mathrm{avant} \mathrm{l'apparition} \mathrm{do} \mathrm{nouvelles}$ constructions au Haut Moyen Âge.

\section{Palais-Grillet (42-46 rue) : $300 \mathrm{~m}^{2}$; FSP (Ayala, DFS,} 1990, 1992).

Période 1 : cailloutis rhodanien (sup. cntre 162,50 et 163,60 m NGF) surmonté par des alluvions du Rhône, datées par ${ }^{14} \mathrm{C}$ de $2205 \pm 105 \mathrm{BP}$ (laboratoire de radiocarbone Lyon I). Période 2 : séquences alluvialcs présentant à leur sommet des traces anthropiques (sup. entre 164 et $164,10 \mathrm{~m} \mathrm{NGF}$ ) ; les plus anciennes, des fosses au nombre de quatrc, se situent aux alentours de l'ère ; les plus récentes, des trous de poteaux et des fragments de sols, à la fin du règne d'Augustc ou au début de cclui de Tibère. Période 3 : dernière phase d'alluvionnement et apparition sous Tibère d'unc occupation étendue à tout le site ; les maçonneries et les sols (sup. vers $164,30 \mathrm{~m} \mathrm{NGF)} \mathrm{sont}$ détruits au milieu du I ${ }^{\text {er }}$ s. apr. J.-C. Période 4 : nouvelles constructions aux fondations puissantes (édifice public?), détruites à la charnière du II $^{\mathrm{c}}$ et du $\mathrm{III}^{\mathrm{e}} \mathrm{s}$. apr. J.-C. (sup. 164,90 m NGF). Période 5 : trous de piquets et fosses, installés dans les niveaux de destruction de la période précédente, attestant une occupation ou une fréquentation du site dans le courant du IV's. apr. J.-C.

12. République (place de la, et rue Childebert) : $2000 \mathrm{~m}^{2}$; FSP (Arlaud et al., DFS, 1992 ; Arlaud et al., 1998).

Rue Childebert : un bras du Rhône, large de près de $22 \mathrm{~m}$, orienté nord-ouest/sud-est, dont le colmatage est postérieur à $5785 \pm 50 \mathrm{BP}(4785-4530 \mathrm{BC}) ;$ un sondage a ćgalement révélé un dépôt d'amphores couchées, scellées par des dépôts sableux, qui témoignent que cette zone est encore un milieu inondable au début du I ${ }^{\mathrm{cr}} \mathrm{s}$. apr. J.-C. (vers $163,60 \mathrm{~m} \mathrm{NGF).}$

Place de la République-Période 1 : substrat caillouteux rhodanien (autour de $163 \mathrm{~m}$ NGF). Période 2 : alternance de dépôts fins et grossiers jusqu'au règne de Claude, au plus tard ; phase 1, les plus récents scellaient, dans la partie nord du site, un fragment de muret et une fosse datés du début de l'c̀re ; phase 2, sous Tibère, après un nouveau débordement du fleuve, du mobilier indique que la partie nord du site est sinon occupée tout au moins fréquentée (sup. entre 163,40 et 163,60 m NGF) ; phase 3, sous Tibère, vestiges d'une occupation plus étendue en direction du sud (caniveau, bassin, lambeaux de sols, fosses et vestiges de trois murs). Période 3 : après plusicurs épisodes de crue, l'occupation se réduit de nouveau à la partic nord du site et se compose uniquement de sols datés du milieu du $\mathrm{I}^{\mathrm{er}} \mathrm{s}$. apr. J.-C. (163-164 m NGF) ; elle est abandonnée sous les Flaviens. Période 4 : au nord du site, quelques fragments de sols et de murs appartenant à une occupation détruite à la fin du $\mathrm{I}^{\mathrm{cr}} \mathrm{s}$. apr. J.-C. ; ses matériaux sont récupérés, peut-être, jusqu'au milieu du $\mathrm{II}^{\mathrm{c}}$ s. apr. J.-C. Période 5 : remblaiement de tout le site soit dans la première moitié du II ${ }^{e} \mathrm{~s}$. apr. J.-C., soit dans le courant du III's. apr. J.-C. (sup. entre 163,30 et 164,70 m NGF) ; aucune 
construction n'est élevée pendant cette pćriode; la date d'abandon du site est inconnue.

13. Célestins (place des) : $2000 \mathrm{~m}^{2}$; FSP (Arlaud et al., DFS, 1993 ; Laubenheimer, Bonnet, 1998).

Période 1 : cailloutis rhodanien (sup. 162,60 m NGF) surmonté d'alluvions fines issues de débordements lents du fleuve. Période 2 : retours réguliers du Rhône et arrivée des premiers flux de la Saône. Période 3 : mobilier atypique fossilisé à l'intérieur de microchenaux ou de fossés creusés par l'homme, le tout scellé par une séquence alluviale de Saône (sup. 163,20 m NGF). Période 4 : sol ćpais du milieu du I ${ }^{\mathrm{cr}}$ s. apr. J.-C., recouvert d'alluvions traduisant une forte intluence du Rhône (sup. $163,50 \mathrm{~m}$ NGF). Période 5 : trous de poteaux sans organisation apparente, fermés par des dépôts de Saône qui contenaient un mobilier du dernier quart du $\mathrm{I}^{\mathrm{er}} \mathrm{s}$. et du début du siècle suivant (sup. 163,60 m NGF). Période 6 (aucun ćpisode de crue) : phase 1 , deux pièces dans la partie sud-est du site dont un des sols est daté de la scconde moitié du II' s. apr. J.-C. (sup. $164,50 \mathrm{~m} \mathrm{NGF}$ ) ; phase 2, creusement d'un fossé orienté estouest, au nord, en avant des deux pièces précédentes; phase 3, abandon du fossé à la fin du $\mathrm{II}^{\mathrm{e}} \mathrm{s}$. apr. J.-C. pour l'aménagement d'un espace ouvert, délimité par un nouvcau fossé de même orientation que le précédent; phase 4, les deux pièces sont dotées de mosaiques. Période 7: campagne de travaux à la fin du II $^{e}$-début III ${ }^{e}$ s. apr. J.-C. étendant l'espace construit au nord, puis exhaussement des sols de la partie nouvellement construite (164,50 m NGF). Période 8 : installation de vides sanitaires composés de fragments ou d'amphores entières dans plusicurs pièces de l'habitat. Période 9: exhaussement général des niveaux et construction d'un caniveau dans le courant du IV $\mathrm{I}$ s. apr.J.-C. ; le site est ensuite occupé entre le VII et le IX`s. apr. J.-C.

14. Hôtel Dieu (rue Bellecordière) : $450 \mathrm{~m}^{2}$; FSU (Jacquin et al., DFS, 1983).

Vestiges très fragmentaires situés entre 164 et $164,50 \mathrm{~m}$ NGF : mur nord-sud correspondant à la façade orientale d'un bâtiment construit au début du $\mathrm{II}^{\mathrm{e}} \mathrm{s}$. apr. J.-C., puis réaménagé au cours de ce siècle ; à une date indéterminée, un second mur double à l'ouest la façade citće ci-dessus.

15. République-Bellecordière ( 83 rue de la) : $75 \mathrm{~m}^{2}$; FSP (Célestin-Le Nézet et al., DFS, 1998).

Période 1 : substrat caillouteux rhodanien (sup. moy. 162,70 m NGF) surmonté d'alluvions sablo-limoneuses (sup. $163,60 \mathrm{~m} \mathrm{NGF}$ ) ; dans ces alluvions, vers $163,30 \mathrm{~m}$ NGF, présence de céramiques augustéennes non roulées. Période 2 : vers 20-40 apr. J.-C. au plus tard, premières constructions élevées à proximité d'un ancien chenal ou d'un chemin bordé d'un fossé (sup. 163,80-164,50 m NGF). Période 3 : entre 40-50 et la fin du $\mathrm{I}^{\text {er }}$ s. apr. J.-C., aménagement d'un chemin bordé d'un fossé et reconstruction des bâtiments de la période précédente (sup. 164,75 m NGF). Période 4 : après destruction de l'état précédent, construction de deux nouveaux bâtiments et comblement partiel du fossé suivi de l'installation, dans les premières décen- nies du II ${ }^{\mathrm{c}}$ s. apr. J.-C., d'un égout en bois dans l'axe de celui-ci (sup. 164,87-164,94 m NGF). Période 5: abandon de l'état antérieur au plus tôt à la fin du II $^{\mathrm{c}}$-III ${ }^{\mathrm{e}}$ s. apr. J.-C. Période 6 : scul le chcmin fait l'objet d'un entretien dans le courant du IV $\mathrm{V}^{\mathrm{c}} \mathrm{s}$. apr. J.-C. ; destruction et abandon du site à la fin du IV ${ }^{\mathrm{c}} \mathrm{s}$. apr. J.-C.

16. Chambonnet (place) : $150 \mathrm{~m}^{2}$; FSP (Ayala, DFS, 1997).

Période 1 : cailloutis déposé par un chenal actif de la Saône (sup. $163 \mathrm{~m} \mathrm{NGF).} \mathrm{Période} 2$ : séquence alluvionnaire à l'intérieur de laquclle s'intercalent les traces d'une fréquentation humaine de la fin du $\mathrm{I}^{\mathrm{er}} \mathrm{s}$. av. J.-C. ou du début du Ir ${ }^{\mathrm{er}}$ s. apr. J.-C. Période 3 : rejets d'activités artisanales sous Tibère. Période 4 : premiers vestiges de constructions à l'époque tibéro-claudienne (vers 164,50 m NGF). Période 5 : construction d'un nouveau bâtiment sous Claude ou Néron dont les pièces, au nord, sont dotées de pavements (vers $165 \mathrm{~m} \mathrm{NGF).} \mathrm{Période} 6:$ modifications du bâtiment précédent au cours du II ${ }^{e}$ s. apr. J.-C. ; destruction et récupération de ses matćriaux à la fin du $\mathrm{IJ}^{\mathrm{e}} \mathrm{s}$. ou au début du III ${ }^{\mathrm{e}}$ s. apr. J.-C. Période 7 : traces fugaces d'occupation au III' s. apr. J.-C.

17. Station Bellecour (place Bellecour). À l'est de la place, sous la station de la ligne D du métropolitain : $16 \mathrm{~m}^{2}$; CS préalable à $17^{\text {bis }}$ (Becker, DFS, 1982).

Période 1 : substrat caillouteux rhodanien. Période 2 : au sommet d'une séquence limoneuse (sup. 163,15 m NGF), deux rangs de huit amphores datées de la fin du $\mathrm{I}^{\mathrm{er}} \mathrm{s}$. apr. J.-C. et un amas de fragments du même contenant.

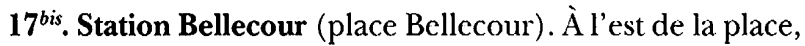
sous la station de la ligne D du métropolitain : $1500 \mathrm{~m}^{2}$; FSP (Burnouf, DFS, 1985 ; Vérot-Bourrély et al., DFS, 1989 ; Burnouf, Laubenheimer, 1998).

Période 1 : substrat caillouteux et dépôts sablonneux rhodaniens recoupés par un microchenal (sup. vers $162 \mathrm{~m} \mathrm{NGF).}$ Période 2 : colmatage progressif du bras avec construction d'un banc renforcé par une levéc de terre et l'installation d'amphores à la fin du I ${ }^{\mathrm{cr}} \mathrm{s}$. apr. J.-C. (sup. vers $163,50 \mathrm{~m} \mathrm{NGF).}$

18. Antonin-Poncet (parking, place) : $3000 \mathrm{~m}^{2}$; FSP (Becker et al., 1989).

Période 1: banc caillouteux (sup. vers 162,50 m NGF) mis en place par le Rhône entre 3500-3600 et 765-400 av. J.-C. (datation corrigée ${ }^{14} \mathrm{C}$ sur deux troncs subfossiles). Période 2 : phase 1 , dépôts sablo-limoneux pollués à l'ouest par quelques débris d'origine anthropique; phase 2, dépôts sablo-limoneux marquant la fin de la construction exclusivement alluviale du site. Période 3 : traces ténues d'une occupation gallo-romaine sous la forme de fosses et de remblais dont le mobilier s'échelonne entre le milieu du $\mathrm{I}^{\text {er }}$ et le III ${ }^{\mathrm{e}}$ s. apr. J.-C. (sup. vers $163,50 \mathrm{~m}$ NGF).

19. Kiosque Bellecour (place Bellecour). Au sud de la place, vis-à-vis de la rue Victor-Hugo : $80 \mathrm{~m}^{2}$; FSP (Becker, DFS, 1983).

Période 1 : sommet d'une séquence alluviale (sup. 162,88 m 
NGF). Période 2 : un mur est-ouest et un sol de tuileau (162,93 m NGF) détruits et remblayés au milieu du $\mathrm{I}^{\mathrm{er}} \mathrm{s}$ apr. J.-C. Période 3: argile sableuse déposée par un débordement de crue du Rhône (sup. $164 \mathrm{mNCF}$ ). Période 4 : denx murs parallèles, nord-sud (ressaut de fondation 163,30 m NGF), recouverts d'une épaisse couche de démolition du début du III ${ }^{\mathrm{e}} \mathrm{s}$. apr. J.-C.

20. Facultés catholiques (25 rue du Plat) : $25 \mathrm{~m}^{2}$; FSP (Monin et al., DFS, 1986).

Période 1 : substrat caillouteux rhodanien surmonté de deux séquences alluvionnaires, la seconde étant consécutive à des débordements de crues de la Saône. Période 2 : fragments de céramique et traces anthropiques de 20-10 av. J.-C. (sup. $163,70 \mathrm{~m} \mathrm{NGF}$ ). Période 3 : un fragment de mur (sup. fondation $164,20 \mathrm{~m} \mathrm{NGF}$ ) et une fosse, datés entre 40 et 60 apr. J.-C. Période 4 : sous Néron, remblaiement du site et construction d'un mur (sup. fondation 164,70 m NGF) élevé sur une aire ouverte, peut-être une voie. Période 5 : nouveaux remblaiements à l'époque flavienne (sup. vers 164,60 m NGF) clos par des niveaux modernes.

21. Sainte-Hélène ( 5 rue) : $40 \mathrm{~m}^{2}$; CS (Monnoyeur-Roussel et al., DFS, 1992b).

Sondage I-Période 1 : remblais composés de matériaux de construction riches en céramiques de la fin de la première moitié du $\mathrm{I}^{\mathrm{er}}$ s. apr. J.-C. (sup. 163,27 m NGF). Période 2 : phase 1, construction d'un mur; phase 2 , reprise du mur précédent et sols (163,93 m NGF), clos par un dépôt sableux, peutêlre une inondation. Période 3 : exhaussement des nive'aux (sup. vers 164,20 m NGF) et reprise du mur de la période précédente; le tout fermé par plusieurs niveaux de démolition (sup. 164,90 m NGF). Période 4 : abandon fin III ${ }^{\mathrm{c}}$, voire début IV's. apr. J.-C.

22. Widor (place) : $70 \mathrm{~m}^{2}$; FSU (Walker, DFS, 1980).

Période 1 : couche argileuse contenant un mobilier abondant (bois, céramiques) du deuxième quart du $\mathrm{I}^{\mathrm{er}} \mathrm{s}$. apr. J.C.C. (sup. 164,24 m NGF). Période 2 : séquence composée d'une démolition et d'une couche cendreuse close par une couche d'argile. Période 3 : une fosse riche en ossements animaux (bœufs, chevaux, etc.), recouverte par un sol (164,57 m NCFF) et scellée par une couche d'argile jaune, peut-être une inondation; aucune évolution des formes céramiques. Période 4 : démolition, zone de rejet ou fosse $(164,96 \mathrm{~m} \mathrm{NGF})$ contenant de nombreux ossements animaux et des fragments d'enduits peints, le tout recouvert par une couche sablcusc, peut-être un débordement de crues. Période 5: deux murs est-ouest construits dans des remblais ( $165,43 \mathrm{~m} \mathrm{NGF)} \mathrm{à} \mathrm{matrice} \mathrm{sableuse}$ qui contenaient des blocs de schiste, des fragments de briques crues et de mortier; dans la seconde moitié du ${ }^{\text {cr }}$ s. apr. J.-C., nouveau remblaiement avec les mêmes types de matériaux que précédemment et construction d'un sol. Période 6 : matériaux de destruction des élévations de la période antérieure et niveaux modernes

\section{LA RIVE DROITE DE LA SAÔNE}

23. Quarantaine I (36-38 rue de la) : $20 \mathrm{~m}^{2}$; CS (Desbat $e t$ al., DFS, 1978).

Trois murs gallo-romains (sup. entre 169,50 et $170,10 \mathrm{~m}$ NGF), recouverts par plusieurs remblais, de la fin du $\mathrm{II}^{\mathrm{e}} \mathrm{s}$./début du III ${ }^{\mathrm{e}} \mathrm{s}$. apr. J.-C., et de la fin du III' $\mathrm{e}$./début IVes. apr. J.-C. ; par ailleurs, de gros blocs de poudingue, pris dans les remblais les plus récents, attestent, à la fin de l'Antiquité, une érosion des balmes qui dominaient le site.

24. Quarantaine II (8 rue de la) : $13 \mathrm{~m}^{2}$; CS (Jacquin et al., DFS, 1981).

Un sol de tuileau $(169,29 \mathrm{~m} \mathrm{NGF})$ et un mur recoupant un épais remblai de démolition (inf. sondage 167,89 m NGF), au plus tôt du $I V^{c}$ s. apr. J.-C.

25. Clément-V (place Benoît-Crépu) : $450 \mathrm{~m}^{2}$; FSP (Monnoyeur-Roussel et al., DFS, 1992a).

Période 1 : substrat formé d'un cailloutis de Saône (sup. moy. 160,40 m NGF), recouvert par des alluvions sablo-limoneuses où se distinguent les influences respectives de la Saône et du Rhône; deux de ces dépôts, l'un de Saône, l'autre une crue majeure du Rhône, conservaient des bois fossiles, datés respectivement aux environs de 50 et 200 av. J.-C. Période 2 : phase 1 , céramiques et structure en blocs de granite (entre 161,80 et $162 \mathrm{~m} \mathrm{NGF)}$ de la première moitié du règne d'Auguste, scellées par un retour de crue de Saône; phase 2, niveaux anthropisés de la première moitié du règne d'Auguste, composés de trous de poteaux et de blocs de granite (entre 162 et 162,50 m NGF). Période 3 : alluvionnement du site sous l'action prédominante de la Saône durant le I ${ }^{\text {er }} \mathrm{s}$. apr. J.-C. (sup. $163,30 \mathrm{~m} \mathrm{NGF}$ ), avec traces de fréquentation épisodique. Période 4 : dans la première moitié du $\mathrm{II}^{\mathrm{e}} \mathrm{s}$. apr. J.-C., remblaiement de la partie centrale, puis traces d'une première occupation à la fin $\mathrm{du} \mathrm{II}{ }^{\mathrm{e}} \mathrm{s}$. apr. J.-C. (trous de poteaux et fosses vers 163,50 m NGF). Période 5 : ensemble de constructions fragmentaires de la fin du II' s. apr. J.-C., abandonné au début du $I^{\mathrm{e}} \mathrm{s}$. apr. J.-C. ; la partie ouest du site enregistre jusque dans le deuxième quart du III's. apr. J.-C. les derniers retours de crues. Période 6 : construction, sur des orientations différentes, d'un nouvcau bâtimcnt au IV ${ }^{\mathrm{e}}$ s. apr. J.-C. (164,50 m NGF) ; celui-ci cst modifié plusieurs fois au cours de son utilisation. Période 7 : destruction du bâtiment précédent, puis traces d'occupation, entre les Ve-VI ${ }^{e}$ s. et le VII ${ }^{e}$ s. apr. J.-C., avant l'abandon du site.

26. Adolphe-Max (avenue) : $1100 \mathrm{~m}^{2}$; FSP (Villedieu, 1990).

Période 1 : banc de gravier rhodanien (sup. vers $162,50 \mathrm{~m}$ NGF), recoupé par un bras secondaire de Saônc. Période 2 : installation d'un bâtiment en rive gauche du bras secondaire dans le troisième quart du $\mathrm{II}^{\mathrm{c}} \mathrm{s}$. apr. J.-C. Période 3 : extension du bâtiment en rive droite dans le deuxième quart ou vers le milieu du III' s. apr. J.-C., puis colmatage définitif du bras. Période 4 : construction de thermes vers la fin du IV ${ }^{\mathrm{e}} \mathrm{s}$. 
apr. J.-C. ou le début du siècle suivant (sup. vers $165 \mathrm{~m} \mathrm{NGF).}$ Période 5 : dans le courant des $\mathrm{V}^{\mathrm{c}}$ et $\mathrm{VI}^{\mathrm{c}}$ s. apr. J.-C., modifications du bâtiment accompagnées d'une régression de la fonction thernale.

27. Funiculaire Saint-Jean (rues Tramassac et J.-Carriès) : $900 \mathrm{~m}^{2}$; FSP (Arlaud el al., 1994).

Période 1 : chenal ancien de la Sâne. Période 2 : installation en rive droitc de pieux en chêne (sup. $160 \mathrm{~m} \mathrm{NGF),} \mathrm{destinés} \mathrm{à}$ soutenir le platelage d'un quai ou d'une voie ; datations dendrochronologiques de trois pieux : 27 av., 8 av., et 18 apr. J.-C. ARC 87/R109D (Archéolabs). Période 3: seconde moitié du $\mathrm{I}^{\mathrm{er}} \mathrm{s}$. apr. J.-C., début du colmatage du chenal sous l'action de courants lents, puis de l'homme. Période 4 : seconde moitié du II' ${ }^{\mathrm{s}}$ s. apr. J.-C., nouvel affaiblissement des courants et réduction de moitié de la largeur du chenal. Période 5 : accélération du processus de colmatage et colmatage définitif des chenaux résiduels par apport de nouveaux remblais au début du $\mathrm{III}^{\mathrm{e}} \mathrm{s}$. apr. J.-C. (sup. vers 163,50 m NGF) ; construction d'un premier bâtiment, peut-être un édifice public, dans la première moitié du III s. apr. J.-C. ; pendant cette période, aménagement, à l'ouest du site, d'une voie dallée nord-sud, dont l'utilisation se prolonge jusqu'à la fin du $V^{\mathrm{c}} \mathrm{s}$. apr. J.-C. ; traces d'occupation à l'est de cette voie. Période 6 : remblaiement général et nivellement du site avant reconstruction.

28. Le Chamarier (37 rue Saint-Jean) : $20 \mathrm{~m}^{2}$; CS (Arlaud, DFS, 1990).

Période 1 : traces anthropiques entre deux couches d'inondation (vers $164,40 \mathrm{~m} \mathrm{NGF).} \mathrm{Période} 2$ : radier de gravier (sup. 164,56 m NGF) contenant de la céramique des III ${ }^{c}-I^{c} \mathrm{~s}$. apr. J.-C. Période 3 : Haut Moyen Âge, mur en pierres liées à la tcrre ct dcux fosses (vers 164,50 m NGF), dont une cuvelée en bois. 1998)

29. Quai Pierre-Scize I $\left(\mathrm{n}^{\circ} 48\right): 80 \mathrm{~m}^{2}$; CS (Ayala et al., DFS,

Murs et niveaux gallo-romains entre $170-173,18 \mathrm{~m}$ (substrat) et 171,80-173,64 m NGF (sup. vestiges) ; seul élément de datation une couche de démolition sous un niveau moderne (II's. apr. J.-C.).

30. Quai Pierre-Scize II (nos 1414 $4^{\text {ter }}$, immeuble sur cour) : $2000 \mathrm{~m}^{2}$; FSU (Lasfargues, DFS, 1982, p. 417).

Ensemble de vestiges antiques, peut-être des entrepôts en bordure de Saône ; aucune chronologie.

31. L'Observance I (ancien couvent de, CEMAGREF, 2 quai Chauvau) : nombre de mètres carrés non communiqué ; FSU (Jacquin).

Vestiges très détruits et fragmentaires : sols en béton de tuileau, caniveaux ; céramiques du IV ${ }^{\mathrm{e}} \mathrm{s}$. apr. J.-C.

32. L'Observance II (ancien couvent de, ENSM, 2 quai Chauvau) : $1400 \mathrm{~m}^{2}$; FSU (Mandy).
Constructions gallo-romaines très arasées; aucune chronologie ; plusieurs sépultures en coffre de dalle du Haut Moyen Âge.

33. Arloing (quai) : $2000 \mathrm{~m}^{2}$; FSP (Tranoy, 1995).

Période 1 : bras ancien de la Saône (sup. vers $160 \mathrm{~m} \mathrm{NGF),}$ surmonté de dépôts issus de processus de colluvionnement (sup. 166,20-165 m NGF). Période 2 : traces de fréquentation du site de peu antérieures aux premières constructions. Période 3 : bâtiments élevés peu avant le milieu du I ${ }^{\mathrm{er}} \mathrm{s}$. apr. J.-C. dans les parties sud-est et nord du site. Période 4 : traces d'une inondation puis remblaiement du site au milieu du $I^{\text {er }}$ s. apr. J.-C. ou dans la seconde moitié de ce siècle au plus tôt ; restructuration des bâtiments existants et apparition des premières sépultures à la fin du $\mathrm{I}^{\mathrm{er}}$-début $\mathrm{II}^{\mathrm{e}} \mathrm{s}$. apr. J.-C. Période 5 : multiplication du nombre des sépultures entre la seconde moitié du II' ${ }^{\mathrm{s}}$ s. et le $I^{\mathrm{e}} \mathrm{s}$. apr. J.-C. ; parmi les constructions dont les niveaux étaient conservés, deux sont abandonnées définitivement, l'une à la fin du III $s$. apr. J.-C. ou au début du siècle suivant, l'autre dans la seconde moitié du IV s. apr. J.-C. Période 6 : abandon du site après le IV ${ }^{\mathrm{e}}$ s. apr. J.-C.

\section{LA PLAINe DE VAISE}

34. Charavay (ZAC, rue du Chapeau rouge) : $2000 \mathrm{~m}^{2}$; FSP (Lascoux, 1994).

Période 1: traces d'une occupation du site vers la fin du $\mathrm{II}^{\mathrm{c}} \mathrm{s}$. av. J.-C. Période 2 : occupation pré-augustéenne et augustéenne. Période 3 : vaste demeure à caractère rural occupée entre 30 av. J.-C. et la fin du règne d'Auguste ou de celui de Tibère. Période 4 : voies est-ouest et nord-sud limitant l'extrémité ouest de deux îlots incomplets ; densification progressive et reconstruction régulière du bâti à l'intérieur des parcelles pendant tout le $\mathrm{I}^{\mathrm{er}} \mathrm{s}$. apr. J.-C. Période 5 : reconstruction presque générale à la fin du I ${ }^{\text {er }}$ s. apr. J.-C. ou au début du siècle suivant, avec création d'un collecteur sous la voie nord-sud. Période 6 : maintien des voies et installation d'un atelier de potier au IVe s. apr. J.-C. et à l'intérieur de l'îlot sud ; la date exacte de l'abandon du site demeure inconnue.

35. Valmy (station de la ligne $\mathrm{D}$ du métropolitain, rue M.-Berthet) : $2500 \mathrm{~m}^{2}$; FSP (Célestin-Le Nézet et al., DFS, 1993).

Période 1 : substrat entre 164,70 et 164,80 m NGF. Période 2 : fréquentation du site, puis construction d'un premier habitat (sols vers $165 \mathrm{~m}$ NGF) dans la seconde moitié du règne d'Auguste. Période 3 : reconstruction de l'habitat précédent et apparition de deux autres bâtiments de même destination dans la première décennie de l'ère. Période 4 : épisode de colluvionnement. Période 5 : apparition d'un découpage parcellaire, campagne de construction et de reconstruction à la fin du règne d'Auguste ou au début de celui de Tibère ; seconde campagne sous Claude au plus tôt. Période 6 : restructuration complète entre la fin du $\mathrm{I}^{\mathrm{er}} \mathrm{s}$. apr. J.C. et la première moitié du siècle suivant; travaux d'assainissement, en particulier remblaiement général du site (sup. moy. 165,50 m NGF) ; agran- 
dissement en fonds de parcelles; nombre de parcelles porté à sept. Période 7 : abandon des constructions et apparition des premières sépultures dans le courant du $\mathrm{III}^{\mathrm{e}}-\mathrm{IV}^{\mathrm{e}} \mathrm{s}$. apr. J.-C. Période 8: sépultures du $\mathrm{V}^{\mathrm{c}}-\mathrm{VI}^{\mathrm{c}} \mathrm{s}$. apr. J.-C.

36. Marietton (rue) : $400 \mathrm{~m}^{2}$; FSP (Monin et al., DFS, 1995).

Période 1 : occupation de l'Âge du Bronze. Période 2 : fossé du Second Âge du Fer (vers 165 m NGF). Période 3 : traces d'une occupation dans la seconde moitié du règne d'Auguste. Période 4 : remblaiement général (vers $165,50 \mathrm{~m} \mathrm{NGF),} \mathrm{recons-}$ truction et abandon au milieu du $\mathrm{I}^{\mathrm{er}} \mathrm{s}$. apr. J.-C. Période 5 : aménagements artisanaux abandonnés dans le dernier quart du $\mathrm{I}^{\mathrm{cr}} \mathrm{s}$. apr. J.-C. Période 6 : nouveau bâtiment construit sur les mêmes orientations que précédemment, suivi d'un arasement du site. Période 7 : traces de fréquentation au plus tôt à la fin du III s. apr. J.-C.

37. Les Blanchisseries (rue du Bourbonnais) : $20 \mathrm{~m}^{2}$; CS et FSP (Tarras, DFS, 1993 ; Bellon et al., DFS, 1993 ; Rahatsöz et al., DFS, 1995).

La partie nord du site est traversée par le talweg d'un cours d'eau actif pendant l'Antiquité ; FSP dans la partie sud du site, la plus densément occupée. Période 1 : fosse du premier tiers du $\mathrm{I}^{\mathrm{er}} \mathrm{s}$. apr. J.-C. et four non daté sur la rive nord d'un bras secondaire. Période 2 : sur la rive gauche de ce bras, couche limoneuse anthropisée, datée entre 80 et 120 apr. J.C. et interprétée comme une zone de rejets. Période 3 : sur la même rive et à proximité du bras, aménagements de quatre fossés successifs, associés ou non à des trous de poteaux, orientés est-ouest et datés entre la seconde moitié du $\mathrm{II}^{2} \mathrm{~s}$. et la fin du $\mathrm{III}^{2} \mathrm{~s}$. apr. J.-C. (sup. entre 164,60 et 165,10 m NGF). Période 4 : fossé orienté nord-sud de la fin III'-IV' s. apr. J.-C. (sup. 165,20 m NGF).

38. Horand (rue du Docteur-) : $18000 \mathrm{~m}^{2}$; CS et FSP (Bellon et al., DFS, 1991, 1992, 1994a et b, 1995) (Horand I à IV).

Période 1: occupation du Bronze ancien et final. Période 2: occupation du Prcmicr Âgc du Fer. Période 3 : fréquentation du site entre le début de l'ère et les Flaviens, caractérisée par la présence de drains, d'une fosse et de deux sépultures du $\mathrm{I}^{\mathrm{er}} \mathrm{s}$. apr. J.-C. (Horand I, II ct III) ; puis sur Horand II uniquement. Période 4 : à la fin du Ier et au début du $\mathrm{II}^{\mathrm{e}} \mathrm{s}$. apr. J.-C., deux incinérations isolées, un tronçon de voirie et deux bassins, peutêtre ccux d'une meuncric hydraulique (zone I) ; un tronçon de voirie, un puits, deux puits-tonneaux et des fragments de meules rotatives basses au nombre de trois au minimum (\%one II) ; d'autres fragments de meule dans l'angle intérieur de deux murs perpendiculaires (CS). Période 5 : en zone I, vestiges d'habitat abandonné dans le courant du II' s. apr. J.-C. ; les zones I et II sont ensuitc recoupécs par des talwegs. Période 6 : à la fin du III et au début du IV e s. apr. J.-C., en zones I et II, traces de constructions sur potcaux de bois. Période 7 : creuscment, postérieurement au IV s. apr. J.C. et en zone I, d'un second talweg.
39. Gorge de Loup I (station de la ligne $\mathrm{D}$ du métropolitain, rue M.-Berthet) : $2500 \mathrm{~m}^{2}$; FSP (Bellon, 1995a ; Burnouf, 1989).

Période 1 : occupation du Premier Âge du Fer (six sépultures, une palissade et les vestiges d'un habitat). Période 2 : traces de fréquentation du site à l'époque augustéenne. Période 3 : bassin (sol à $183 \mathrm{~m} \mathrm{NGF)} \mathrm{et} \mathrm{caniveau} \mathrm{de} \mathrm{bois} \mathrm{de} \mathrm{la} \mathrm{fin}$ du premier quart du $I^{\text {er }}$ s. apr. J.-C.; autre bassin en bois du milieu du ${ }^{\mathrm{Ir}}$ s. apr. J.-C. ; cinq sépultures isolées. Période 4 : partie d'un grand bâtiment, non daté. Période 5 : construction, dans la cour du bâtiment antérieur, d'une batterie de bassins dans le dernier quart du $\mathrm{I}^{\mathrm{er}}$ s. apr. J.-C. (sols vers $283,50 \mathrm{~m}$ NGF) ; abandon début du II ${ }^{\mathrm{s}}$ s. apr. J.-C. Période 6 : traces de fréquentation du site aux $\mathrm{II}^{\mathrm{e}}-\mathrm{III}^{\mathrm{e}} \mathrm{s}$. apr. J.-C.

40. Gorge de Loup II (gare interroutière, rues M.-Berthet et Professeur-Guérin) : $16000 \mathrm{~m}^{2}$; CS et FSU (Jacquin et al., DFS, 1989 ; Plassot et al., DFS, 1991b).

Période 1 : traces ténues d'une occupation protohistorique. Période 2 : partie d'un bâtiment du $\mathrm{I}^{\mathrm{er}}$ s. apr. J.-C.

41. Saint-Pierre (ZAC du quarticr, ruc M.-Berthet, ancienne usine Rhodiacéta) : $10000 \mathrm{~m}^{2}$; CS et FSP (Chastel et al., 1995 ; Plassot, 1995).

Période 1: chemin creux ou fossé en picd de versant, peut-être protohistorique. Période 2 : au-dessus de la structure précédente, premier état de la chaussée de la voie de l'Océan (fin du $\mathrm{I}^{\mathrm{cr}} \mathrm{s}$. av. J.-C.). Période 3 : ćlargissement de la chaussée de la voie précédente dans la première décennie du $\mathrm{I}^{\mathrm{er}} \mathrm{s}$. apr. J.-C. Période 4 : à l'ouest de la voie, maison dite aux Xenia (sols 184,50-185,15 m NGF), occupée entre Tibère et le début du règne des Flaviens ; le long de la voie, sépultures (2) de la seconde moitié du I ${ }^{\mathrm{er}} \mathrm{s}$. apr. J.-C. Période 5 : ibidem, sépultures (5) des $\mathrm{II}^{\mathrm{c}}$-III' $\mathrm{s}$. apr. J.-C. Période 6 : rccouvrement progressif de la voie par des processus de colluvionnement et abandon de celle-ci dans le courant du IV s. apr. J.-C., au plus tôt.

\section{LA RIVE GAUCHE DU RHÔNE}

42. Chevrier (rue Père-) : $1000 \mathrm{~m}^{2}$; FSP (Rahatsöz et al., DFS, 1997).

Période I : substrat caillouteux rhodanien (sup. vers $162,83 \mathrm{~m} \mathrm{NGF}$ ), surmonté de recouvrements sableux, puis argileux. Période 2 : traces de fréquentation au Bronze final (vers 163,73 m NGF). Période 3 : creusement d'un fossé large de $5 \mathrm{~m}$ au minimum, profond de $1,5 \mathrm{~m}$. Période 4 : installation d'une voie, sur le bord droit du fossé (inf. 164,56 m NGF) dont les recharges (sup. 164,82 m NGF) contenaient un mobilier appartenant à un horizon des $\mathrm{I}^{\mathrm{er}}-\mathrm{II} \mathrm{e}^{\mathrm{e}} \mathrm{s}$. apr. J.C. ; comblement du fossé à partir du milieu du II ${ }^{\mathrm{s}} \mathrm{s}$. apr. J.-C. ; une incinération secondaire recoupant le comblement du fossé. Période 5 : à l'emplacement du fossé, deux maçonneries, six inhumations et un bloc épigraphique funćrairc de la premic̀re moitić du III s. apr. J.-C. Période 6: dans le courant du $\mathrm{IV}^{\mathrm{c}}$ s. apr. J.-C., traces d'occupation en bordure de la voie. 


\section{ANNEXE II}

\section{DRAINS ET VIDES SANITAIRES D'AMPHORES ET EPAVES (fig. 25)}

\section{DRAINS ET VIDES SANITAIRES}

1. Sans autre précision, à Saint-Nizier (Steyert, 1895, p. 282).

2. 19, rue de la République, « sous toute la superficie de la maison, de la rue Gentil à la place de la Bourse et à 3,50 m de profondeur, un important dépôt d'amphores disposées très régulièrement la pointe en haut. " (Audin, 1956, p. 130-131).

3. Place de la Bourse, à l'angle de la rue de la République, un gisement d'amphores (Lancel, 1975, p. 550). "Cinq amphores dans la vase et du sable, dont une disposée le col en bas » (Turcan, 1980, p. 88, note 11).

4. À l'intersection des rues Dubois et de l'Impératrice (actuelle rue Édouard-Herriot), à $5 \mathrm{~m}$ de profondeur, le gravier du Rhône sur lequel reposait une certaine quantité d'amphores vinaires, rompues à la base ; elles étaient " recouvertes de limon et de terre de remblai " (Boissieu, 1864, p. 33; MartinDaussigny, 1853, p. 15, note 1).

5. Saint-Bonaventure, à douze pieds de profondeur, sur la place où l'on a depuis élevé le presbytère de Saint-Bonaventure, " des amphores renversées reposant sur le sable du Rhône " (Boissieu, 1864, p. 34 ; cf. également Artaud, 1846, p. 145).

6. Croisement du passage de l'Argue et de la rue ÉdouardHerriot ; dépôt d'amphores assimilé à la cargaison d'une épave (Allmer, Dissard, 1889, p. 333).

7. À l'angle de la rue Mercière, lors des fouilles pratiquées pour les fondations du passage de l'Argue et l'établissement de la rue de la Préfecture, « des tuiles, des dépôts d'amphores, des médailles du temps d'Antonin et le sable de la Saône à 15 pieds de profondeur" (Boissieu, 1864, p. 34 et note 3, cf. de même Artaud, 1846, p. 142). On rencontre également la localisation " dans l'angle nord de la place des Jacobins, près de la rue de l'Ancienne-Préfecture »(Turcan, 1980, p. 69).

8. Rue Childebert, lors de la construction du parc de stationnement, gisement d'amphores du début du ${ }^{\mathrm{er}} \mathrm{s}$. apr. J.-C., couchées et scellées par des dépôts sableux du Rhône ( $c f$. annexe I, $\mathrm{n}^{\circ}$ 12, p. 62-63 ; Arlaud et al., DFS, 1992 ; Arlaud el al., 1998).

9. Place des Célestins, lors de la construction du parc de stationnement, à l'intérieur de plusieurs pièces d'un bâtiment occupé au III ${ }^{\mathrm{e}}$ et au IV $\mathrm{V}^{\mathrm{e}} \mathrm{s}$. après J.-C., vides sanitaires constitués d'amphores entières ou fragmentées ( $c f$ annexe I, $\mathrm{n}^{\circ} 13$, p. 63 ; Arlaud $e$ al., DFS, 1993 ; Laubenheimer, Bonnet, 1998).
10. 7, rue Simon-Maupin, amphores à $3,50 \mathrm{~m}$ de profondeur (Audin, 1956, p. 135).

11. Place Le Viste, plusieurs dizaines d'amphores en assainissement à $4 \mathrm{~m}$ de profondeur (Boucher, 1977, p. 483). Au nord-est de la place Bellecour, un autre gisement de plusieurs amphores, vraisemblablement à proximité du précédent (Lancel, 1975, p. 550).

12. Parc de stationnement Bellecour, « 3000 amphores environ, à $3 \mathrm{~m}$ de profondeur, qui formaient un gisement épais de trois lits d'amphores" (Turcan, 1980, p. 77).

13. Bellecour, face à la rue Victor-Hugo, à $4 \mathrm{~m}$ de profondeur, gisement d'amphores couchées (Boucher, 1977, p. 483 ; $c f$. également illustration, inventaire et datation [période augustéenne], in: Burnouf, Laubenheimer, 1998, p. 177).

14. Immeuble à l'angle des rues Victor-Hugo, Sala et Boissac, « un certain nombre d'amphores dans les terrassements (Audin, 1956, p. 134).

15. Lors de la construction de la caserne, rue Sala, « à dix pieds de profondeur, gravier du Rhône [...] ; sur ce gravier des lignes d'amphores vinaires qui se retrouvent un peu partout à l'entour »(Boissieu, 1864, p. 38).

16. Croisement des rues Sala et de la Charité (Hôtel des Finances), «un sol antique isolé par [huit] jarres renversées, tangentes entre elles et disposées en ligne »(Guey, 1958, p. 359).

17. Rues Victor-Hugo (30) et Jarente (29). Non loin de la mosaïue du Cirque, " dans une cave antique, amphores en nombre considérable et arrangées avec soin »(Steyert, 1895, p. 283).

18. Place Ampère, "lit d'amphores sphériques allongées" (Boucher, 1977, p. 485) ; N.d.A. : amphores Dressel 20.

19. Au sud de Saint-Martin-d'Ainay, d'après un dessin de M. l'abbé Pallière, vicaire d'Ainay, trois amphores alignées, disposées col en bas (Steyert, 1895, p. 576, fig. 767).

20. Square Jammot, des amphores auraient été aperçues sous le couvent des Clarisse (angle nord-est du square) (Turcan, 1980, p. 83, note 19). Peut-être également, à cet cndroit ou à proximité, « dcs caves remplics d'amphores, apcrçues lorsqu'on a construit le nouveau couvent de SainteClaire (Artaud, 1846, p. 146). 
ÉPAVEs

A. Sous le central Franklin (rue Franklin), « la membrure d'une grande barque romaine, échouée perpendiculairement à l'ancien rivage, $[. .] ».($ Audin, 1956, p. 13).
B. Restes d'une épave sous Le César, place Tolozan ( $c f$. annexe I, $n^{\circ} 7$, p. 61 ; Becker, Rieth, 1995).

\section{ANNEXE III}

\section{OCCUPATIONS OU TRACES D'OCCUPATION DU IV S. APRÈS J.-C.}

\section{Avertissement}

Les intcrprétations et les datations sont données sous réscrve de modifications qui pourraient être apportées par les auteurs au moment de la publication des résultats.

\section{LES SITES}

1. Saint-Vincent : bâtiment thermal du secteur III utilisé au $I^{\text {e }}$ s. apr. J.-C., voire jusqu'au V $V^{\mathrm{c}} \mathrm{s}$. apr. J.-C. Secteur I, grande demeure (domus) portant les traces d'une occupation au IVe $s$. apr. J.-C. ( $c f$. annexe I, $\mathrm{n}^{\circ} 1$, p. 60).

2. Îlot Vieille-Monnaie : abandon de l'habitat au début du $\mathrm{IV}^{\mathrm{e}} \mathrm{s}$. apr. J.-C., puis occupation sporadique du site au milieu du $\mathrm{IV}^{\mathrm{e}}$ s. apr. J.-C. ( $c f$. annexe I, n ${ }^{\circ}$ 3, p. 60).

3. Terreaux : bâtiment du $\mathrm{N}^{\mathrm{re}} \mathrm{s}$. détruit à la fin du $\mathrm{V}^{\mathrm{c}} \mathrm{s}$. apr. J.-C., ainsi que quelques ensembles fragmentaires rattachés à la même période ( $c f$. annexe $\mathrm{I}, \mathrm{n}^{\circ} 4$, p. 60).

4. Bourse : après abandon des constructions de la période antérieure à la fin du III' s. /début du $\mathrm{IV}^{\mathrm{e}} \mathrm{s}$. apr. J.-C., des trous de potcau ct des murs de galets témoignent d'une occupation dans la partie ouest du site, au IV ${ }^{\text {ve }}$ s. apr. J.-C. ( $c f$. annexe I, $n^{\circ} 9$, p. 62).

5. Palais-Grillet : des trous de poteau et des fosses attestent une occupation ou une fréquentation du site dans le courant du IV's. apr. J.-C. ( $c f$. annexe I, $\mathrm{n}^{\circ} 11$, p. 62).

6. Îlot 24 : fragments de sol en terre battue du IVe s. apr. J.-C. dans le bâtiment abandonné au milieu du IVes. apr. J.-C., puis nouvelles constructions au Haut Moyen Âge (cf. annexe I, $\mathrm{n}^{\circ} 10$, p. 62).

7. Célestins : exhaussement des niveaux du bâtiment et construction d'un caniveau dans le courant du IV s. apr. J.-C. Le site est occupé ensuite entre le VII ${ }^{\mathrm{c}}$ s. et le IX' $\mathrm{IX}^{\mathrm{c}}$. apr. J.-C. ( $c f$. annexe $\mathrm{I}, \mathrm{n}^{\circ} 13$, p. 63).

8. Sainte-Hélène : aban3don fin du $\mathrm{III}^{\mathrm{c}} \mathrm{s}$, , voire début du IV s. apr. J.-C. (cf. annexe I, n² 21, p. 64).
9. Adolphe-Max : le bâtiment du deuxième quart du $\mathrm{III}^{\mathrm{e}} \mathrm{s}$. ou du milieu du III's. apr. J.-C. est maintenu jusqu'au IV ${ }^{\mathrm{e}} \mathrm{s}$. apr. J.-C. Construction de thermes à la fin du IV's. apr. J.-C. qui changent de destination après avoir été restructurés dans le courant du Ve s. et au V'c s. apr. J.-C. Le sitc cst cnsuite occupé au cours du Haut Moyen Âge ( $c f$. annexe I, n² 26, p. 64-65).

10. Funiculaire Saint-Jean : nouvelle voie construite à la fin du III's./début du IV's. apr. J.-C. Entre le IV's. et le VI ${ }^{\mathrm{e}} \mathrm{s}$. apr. J-C., le site continue à être occupé : traces successives d'occupation (fragments de construction, sols, niveaux d'incendie), remblais, rejets de boucherie. L'occupation du site sc poursuit au VI' s. apr. J.-C. ( $c f$. annexe I, n 27, p. 65).

11. Clément-V : abandon des constructions antérieures au début du $\Gamma^{\mathrm{c}} \mathrm{s}$. apr. J.-C., suivi par la construction d'un nouveau bâtiment établi sur des orientations différentes. Ce dernier est modifié à plusieurs reprises. Après son abandon, traces d'occupation du $\mathrm{V}^{\mathrm{e}} / \mathrm{VI}^{\mathrm{e}}$ s. et du VII ${ }^{\mathrm{e}}$ s. apr. J.-C. ( $c f$. annexe I, $\mathrm{n}^{\circ} 25$, p. 64).

12. Quarantaine : sol de tuileau (bassin ?) construit au plus tôt au IV $\mathrm{IV}^{\mathrm{e}}$ s. apr. J.-C. (cf. annexe I, n ${ }^{\circ} 24$, p. 64).

13. Chamarier : fragments de sols, remblais ct niveaux d'incendie du III ${ }^{e}$ s. $/ \mathrm{IV}^{\mathrm{e}} \mathrm{s}$. apr. J.-C. Le site est occupé également pendant le Haut Moyen Âge ( $c f$. annexe I, $\mathrm{n}^{\circ}$ 28, p. 65).

14. Observance : mobilier du IV s. apr. J.-C. ( $c f$. annexe I, $\mathrm{n}^{\circ} 31$, p. 65).

15. Arloing : sur les trois bâtiments construits antérieurement, deux sont abandonnés à la fin du III ${ }^{c} s$-début $\mathrm{IV}^{\mathrm{e}} \mathrm{s}$. apr. J.-C. Le troisième est encore utilisé dans la seconde moitié du $\mathrm{IV}^{\mathrm{e}}$ s. apr. J.-C. Au IV se apr. J.-C., la z.one funéraire continue à être utilisée. Abandon général du sitc apr. lc IVes. apr. J.-C. ( $c f$. annexe I, $\mathrm{n}^{2} 33$, p. 65).

16. Charavay : les éléments de la trame sont encore en place au IVes. apr. J.-C. Toutefois, seul un atelier de potier est attesté à l'intéricur de l'un des deux îlots pendant cette période. La date d'abandon du site est inconnue (cf. annexe $\mathrm{I}, \mathrm{n}^{\circ} 34$, p. 65).

17. Valmy : nécropole des III ${ }^{\mathrm{c}} / \mathrm{IV}^{\mathrm{c}}$ s. apr. J.-C. ( $c f$. annexe I, $\mathrm{n}^{\circ} 35$, p. 65-66). 
18. Saint-Pierre : recouvrement et abandon de la voie de l'Océan dans le courant du IVe s. apr. J.-C., puis occupation au Haut Moyen Âge ( $c f$. annexe I, $n^{\circ} 41$, p. 66).

19. Horand : diverses traces d'une occupation non identifiée à la fin du III ${ }^{e}$ s./début du IV s. apr. J.-C. ( $c f$. annexe I, $n^{\circ} 38$, p. 66).

20. Blanchisseries : un fossé daté de la fin du $1 \mathrm{II}^{\mathrm{e}} \mathrm{s}$./début $\mathrm{IV}^{\mathrm{c}}$ s. apr. J.-C. ( $c f$. annexe I, $\mathrm{n}^{\circ} 37$, p. 66).
21. Chevrier : traces d'occupation et nombreuses fosses datées du IV e s. apr. J.-C. ( $c f$. annexe I, nº 42, p. 66).

\section{LES MOSAÏQUES DU IV' S. APRÈS J.-C.}

A. 3, place Sathonay : seconde moitié du III ${ }^{e}$ ou IV's. apr. J.-C. (Stern, 1967, n 136).

B. 3, rue Jarente : IVe-Ve s. apr. J.-C. (Stern, 1967, n 100).

C. 4 , rue Jarente : tardive (Stern, 1967, $\mathrm{n}^{\circ}$ 102). 